\title{
TYPE Ib/c SUPERNOVAE IN BINARY SYSTEMS. I. EVOLUTION AND PROPERTIES OF THE PROGENITOR STARS
}

\author{
S.-C. Yoon ${ }^{1,2}$, S. E. Woosley ${ }^{1}$, AND N. LANGER ${ }^{2,3}$ \\ ${ }^{1}$ UCO/Lick Observatory, Department of Astronomy \& Astrophysics, University of California, Santa Cruz, CA 95064, USA \\ 2 Argelander Institut für Astronomie, University of Bonn, Auf dem Hügel 71, D-53121, Bonn, Germany \\ 3 Astronomical Institute, Utrecht University, Princetonplein 5, 3584 CC, Utrecht, The Netherlands \\ Received 2010 April 1; accepted 2010 October 5; published 2010 November 22
}

\begin{abstract}
We investigate the evolution of Type $\mathrm{Ib} / \mathrm{c}$ supernova ( $\mathrm{SN} \mathrm{Ib} / \mathrm{c}$ ) progenitors in close binary systems, using new evolutionary models that include the effects of rotation, with initial masses of $12-25 M_{\odot}$ for the primary components, and of single helium stars with initial masses of 2.8-20 $M_{\odot}$. We find that, despite the impact of tidal interaction on the rotation of primary stars, the amount of angular momentum retained in the core at the presupernova stage in different binary model sequences converges to a value similar to those found in previous single star models. This amount is large enough to produce millisecond pulsars, but too small to produce magnetars or long gammaray bursts. We employ the most up-to-date estimate for the Wolf-Rayet mass-loss rate, and its implications for $\mathrm{SN} \mathrm{Ib/c} \mathrm{progenitors} \mathrm{are} \mathrm{discussed} \mathrm{in} \mathrm{detail.} \mathrm{In} \mathrm{terms} \mathrm{of} \mathrm{stellar} \mathrm{structure,} \mathrm{SN} \mathrm{Ib/c} \mathrm{progenitors} \mathrm{in} \mathrm{binary} \mathrm{systems}$ at solar metallicity are predicted to have a wide range of final masses up to about $7 M_{\odot}$, with helium envelopes of $M_{\mathrm{He}} \simeq 0.16-1.5 M_{\odot}$. Our results indicate that, if the lack of helium lines in the spectra of SNe Ic were due to small amounts of helium (e.g., $M_{\mathrm{He}} \lesssim 0.5$ ), the distribution of both initial and final masses of SN Ic progenitors should be bimodal. Furthermore, we find that a thin hydrogen layer $\left(0.001 M_{\odot} \lesssim M_{\mathrm{H}} \lesssim 0.01 M_{\odot}\right)$ is expected to be present in many SN Ib progenitors at the presupernova stage. We show that the presence of hydrogen, together with a rather thick helium envelope, can lead to a significant expansion of some $\mathrm{SN}$ Ib/c progenitors by the time of supernova explosion. This may have important consequences for the shock break-out and supernova light curve. We also argue that some SN progenitors with thin hydrogen layers produced via Case $\mathrm{AB} / \mathrm{B}$ transfer might be related to Type IIb supernova progenitors with relatively small radii of about $10 R_{\odot}$.
\end{abstract}

Key words: binaries: close - stars: evolution - stars: massive - stars: rotation - supernovae: general

Online-only material: color figures

\section{INTRODUCTION}

It is generally believed that Type Ib and Type Ic supernovae result from core-collapse events of naked helium stars. The helium stars are thought to be produced by the loss of the hydrogen envelope, via stellar winds mass loss from massive single stars or via mass transfers in close binary systems.

According to recent stellar models adopting the most upto-date stellar winds mass-loss rates (Meynet \& Maeder 2003, 2005; Eldridge \& Vink 2006; Limongi \& Chieffi 2006; Georgy et al. 2009), the final masses $\left(M_{\mathrm{f}}\right)$ of helium stars produced by mass-losing single stars appear to be too massive to produce typical $\mathrm{SNe} \mathrm{Ib} / \mathrm{c}$ (i.e., $M_{\mathrm{f}}>10 M_{\odot}$ at solar metallicity). Although the limiting mass for black hole $(\mathrm{BH})$ formation is not yet well determined, given their high binding energy, such massive progenitors of $M_{\mathrm{f}}>10 M_{\odot}$ are likely to form BHs, producing faint supernovae or no supernova at all (cf. Fryer 1999). Although very bright SNe Ib/c like SN 1998bw could be produced from such massive helium stars if, for example, powered by rapid rotation (e.g., Woosley 1993; Burrows et al. 2007), such events are shown to be rare (e.g., Podsiadlowski et al. 2004; Guetta \& Della Valle 2007).

By contrast, helium stars with a wide range of masses $\left(2.0 M_{\odot} \lesssim M_{\mathrm{He}} \lesssim 25 M_{\odot}\right)$ can be made from 12 to $60 M_{\odot}$ primary components in close binary systems via the so-called Case A/B mass transfer. ${ }^{4}$ Many of them may end their life as

4 Case A, B, or C mass transfer denotes mass transfer from the primary star during core hydrogen burning, helium core contraction/beginning of core helium burning, or core helium burning and later stages, respectively. On the bright $\mathrm{SNe} \mathrm{Ib} / \mathrm{c}$ leaving neutron stars as remnants, if their final masses are less than about $7-10 M_{\odot}$. Population studies indeed show that close binary stars can produce a sufficient number of $\mathrm{SNe} \mathrm{Ibc}$ to explain their observed rate, without the need of invoking single star progenitors (e.g., Podsiadlowski et al. 1992; de Donder \& Vanbeveren 1998; Eldridge et al. 2008). Therefore, it is most likely that the majority of typical SNe Ibc are produced in binary systems.

The observational evidence for the connection between $\mathrm{SNe}$ Ibc and long gamma-ray bursts (GRBs) has particularly motivated many observational studies to better understand SNe Ibc since the last decade (see Woosley \& Bloom 2006, for a review). Theoretical stellar models of SNe Ibc progenitors are thus highly required nowadays. The most comprehensive studies on the detailed characteristics of SNe Ibc progenitors in binary systems were conducted by Woosley et al. (1995, hereafter WLW95) using mass-losing pure helium star models, and by Wellstein \& Langer (1999, hereafter WL99) using self-consistent binary star models. Although more recent theoretical studies on SNe Ibc progenitors in binary systems can be found in the literature, they have been focused on long GRB progenitors or stellar populations, rather than on the detailed nature of typical SNe Ibc progenitors (e.g., Brown et al. 2000; Izzard et al. 2004; Petrovic

other hand, if mass transfer occurs during helium core contraction/beginning of core helium burning from a star that has already undergone Case A mass transfer, such a mass transfer phase is called Case AB. Case ABB or Case $\mathrm{BB}$ mass transfer denotes mass transfer from the primary star during core helium burning and/or later stages, which has already undergone Case $\mathrm{AB}$ or Case B mass transfer, respectively. 
et al. 2005b; Cantiello et al. 2007; van den Heuvel \& Yoon 2007; Detmers et al. 2008; Eldridge et al. 2008).

In this paper, we revisit the problem of $\mathrm{SNe}$ Ibc progenitors in close binary systems using both binary star and single helium star models up to the neon burning stage, with updated physics of two important ingredients. One is rotation, which was not considered in WLW95 and WL99, and the other is the massloss rate of Wolf-Rayet (WR) stars (Section 2).

This paper is organized as follows. In Section 2, we briefly review recent developments of stellar evolution models regarding the effects of rotation and the WR star mass-loss rate, arguing for the need of updated physics in binary star models. Our adopted physical assumptions and numerical method are discussed in Section 3. In the following section (Section 4), using our binary star evolution models including the effect of rotation and the transport of angular momentum due to hydrodynamic instabilities and magnetic torques, we explore the role of tidal interaction and mass transfer in the redistribution of angular momentum in primary stars. In Section 5, the nature of SNe Ibc progenitors is investigated in terms of final masses, masses of helium and hydrogen layers, radii and mass-loss rates at the presupernova stage, assuming that these properties do not significantly change from neon burning to core collapse. For this purpose, we also present mass-losing single helium star models as a complement to our binary star models, given that the parameter space explored with our binary model sequences is limited. We conclude the paper by discussing observational implications of our results, in Section 6.

\section{ROTATION AND WOLF-RAYET WINDS}

\subsection{Rotation}

Rotation has particular roles in the evolution of massive stars as it changes the stellar structure, induces chemical mixing, and enhances mass loss due to stellar winds (Maeder \& Meynet 2000; Heger et al. 2000). During the last decade, several authors have calculated massive star models up to the pre-supernova stage, considering the redistribution of angular momentum and chemical species due to rotationally induced hydrodynamic instabilities, such as Eddington-Sweet circulations, and the shear instability (Heger et al. 2000; Hirschi et al. 2004). Although these models could explain some observational aspects such as surface abundances of $\mathrm{CNO}$ elements of massive stars and WR star populations at different metallicities (e.g., Maeder \& Meynet 2000; Heger \& Langer 2000; Meynet \& Maeder 2005), their adopted angular momentum transport mechanisms turned out to be too inefficient to explain the observed spin rates of stellar remnants (Suijs et al. 2008). That is, their models predict nearly 2 orders of magnitude higher spin rates of white dwarfs and young neutron stars than the observed ones. These models also imply that almost all WR stars can retain enough angular momentum to produce GRBs, either by magnetar or collapsar formation depending on the final mass. This gives a nearly 1000 times higher ratio of GRBs to SNe than the observationally implied value. On the other hand, Spruit $(1999,2002)$ suggested that magnetic torques resulting from dynamo actions in differentially rotating radiative layers (the so-called Spruit-Tayler dynamo) should be the dominant angular momentum transport mechanism compared to the pure hydrodynamic instabilities. Recent magnetic models that adopt the Spruit-Tayler dynamo according to the prescription by Spruit (2002) are indeed more consistent with observations in terms of the spin rates of stellar remnants (Heger et al. 2005; Suijs et al. 2008). Magnetic mod- els also better explain the fact that long GRBs are rare events compared to normal core-collapse supernovae. Although the Spruit-Tayler dynamo mechanism is still subject to many uncertainties (Denissenkov \& Pinsonneault 2007; Zahn et al. 2007), these recent studies indicate that an efficient angular momentum transport mechanism comparable to what the Spruit-Tayler dynamo predicts is needed to understand the observations.

The above discussion is based on single star models, and the role of rotation in the evolution of massive binary stars remained relatively unexplored. Massive stars in close binary systems are supposed to experience an exchange of mass and angular momentum via mass transfer and tidal interaction, and thus the evolution of binary stars is more complex than that of single stars. Wellstein (2001) and Langer et al. (2003) presented, for the first time, self-consistent calculations of massive binary star evolution models including many relevant effects of rotation and binary interactions: the change of the stellar structure due to the centrifugal force, transport of angular momentum and chemical species due to rotationally induced hydrodynamic instabilities, tidal interaction, transfer of mass from the primary, accretion of mass and angular momentum of the secondary, and resulting changes of the orbit. Their work indicates that the secondary can be easily spun up by mass accretion even up to critical rotation, thus modulating the mass accretion efficiency by the interplay of the enhanced mass loss due to rotation from the spun-up secondary and the mass transfer from the primary. This effect provides important clues to better understand the evolutionary paths of some observed X-ray and WR star binary systems as discussed by Langer et al. (2003) and Petrovic et al. (2005a). Their non-magnetic models also show that massive stars may end up with different core spin rates depending on the history of mass loss/gain during binary evolution, that could be related to the observational diversity of core-collapse supernovae. More recently, Petrovic et al. (2005b) and Cantiello et al. (2007) included the Spruit-Tayler dynamo in their binary models and discussed possible evolutionary paths of massive binary stars toward long GRBs. In this paper, we present new evolutionary calculations of magnetic (i.e., the Spruit-Tayler dynamo is included) massive binary stars for a large parameter space, focusing on the evolution of the primary stars to investigate the nature of typical SNe Ibc progenitors.

\subsection{Mass Loss due to Wolf-Rayet Winds}

WLW95 employed the mass-dependent mass-loss rate of WR stars of Langer (1989) for their helium star models, and WL99 used those of Hamann et al. (1982) and Hamann et al. (1995). Both studies concluded that the final masses of SNe Ibc progenitors in binary systems should converge to a limited range of 2.2-3.6 $M_{\odot}$, even for an initial helium star mass of $20 M_{\odot}$. Later developments of the WR wind theory considering clumpies pointed out, however, that the WR mass-loss rates used by WLW95 and WL99 are significantly overestimated (Hamann \& Koesterke 1998; Nugis \& Lamers 2000). For example, Figure 1 shows that, for helium stars on the zeroage main sequence (ZAMS), the mass-loss rate given by Nugis \& Lamers (2000) is almost an order of magnitude lower than that of Hamann et al. used in WL99. The most recent theoretical models of WR winds by Vink \& de Koter (2005) and Gräfener \& Hamann (2008) also give WR mass-loss rates compatible to the Nugis \& Lamers rate (cf. Figure 1 in Yoon \& Langer 2005).

The reduced WR mass-loss rates have been considered in many recent massive single star models (e.g., Meynet \& Maeder 2003, 2005; Eldridge \& Vink 2006), suggesting large final 


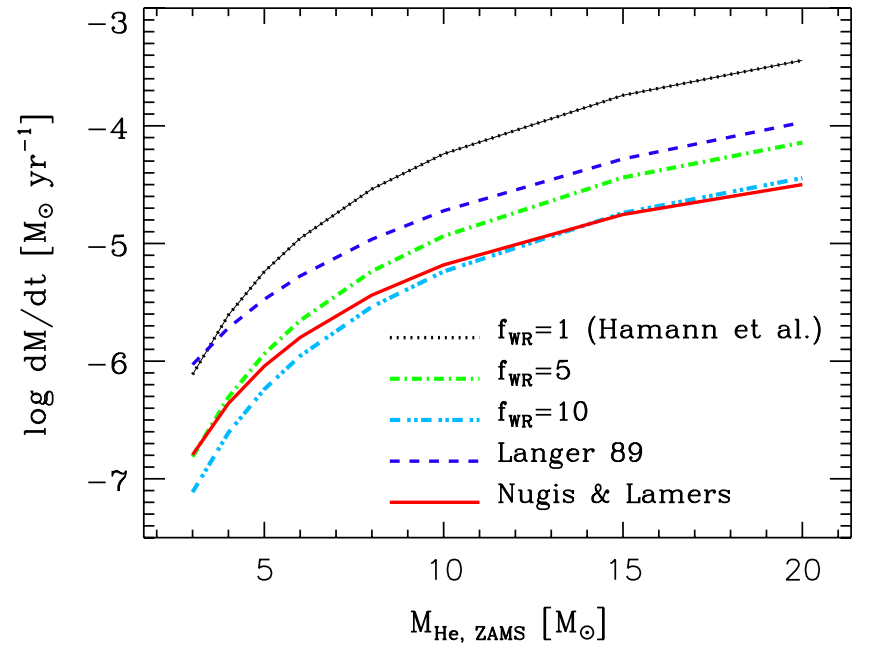

Figure 1. WR mass-loss rates from helium stars on the zero-age main sequence (ZAMS) at solar metallicity. The mass-loss rates of Hamann et al. (see Equation (1)), Langer (1989), and Nugis \& Lamers (2000) are given by dotted, dashed, and solid lines, respectively. The dot-dashed and triple-dot-dashed lines denote the Hamann et al. rates divided by a factor of 5 and 10, respectively (i.e., $f_{\mathrm{WR}}=5$ and 10 in Equation (1)).

(A color version of this figure is available in the online journal.)

masses of WR stars at the presupernova stage $\left(M_{\mathrm{f}}>10 M_{\odot}\right.$ at solar metallicity) compared to $\sim 4 M_{\odot}$ found by Woosley et al. (1993). As argued in the introduction, this leads to the interesting conclusion that most core-collapse events occurring in single WR stars should not produce bright supernovae, rendering the binary star channel even more important for SNe Ibc. Surprisingly, the effect of the new WR mass-loss rate on SNe Ibc progenitors in close binary systems has not been much discussed or largely overlooked. To our knowledge, Pols \& Dewi (2002) are the only authors who addressed this issue in detail. Although WL99 also presented some model sequences with a reduced WR mass-loss rate, their adopted reduction factor was rather modest (two times smaller than the Hamann et al. rate). Here we consider the Hamman et al. WR mass-loss rate reduced by factors of 5 and 10, which reflects the most recent result as discussed above.

\section{NUMERICAL METHOD AND PHYSICAL ASSUMPTIONS}

We have used the same stellar evolution code as in Cantiello et al. (2007), that follows the simultaneous evolution of the two stellar components of a binary system. The effect of the centrifugal force on stellar structure is considered following Endal \& Sofia (1976). The transport of angular momentum and chemical elements is treated as diffusion considering Eddington Sweet circulations, the shear instability, the Goldreich-Schubert-Fricke instability, and the Spruit-Tayler dynamo, as described in Petrovic et al. (2005b). We use $\alpha_{\text {SEMI }}=1.0$ for the semi-convection parameter (Langer et al. 1983), as in Yoon et al. (2006).

The stellar winds mass-loss rate is determined following Kudritzki et al. (1989) with a metallicity scaling of $\left(Z / Z_{\odot}\right)^{0.69}$ (Vink et al. 2001) for the main-sequence phase. WR wind massloss rates are computed according to Hamman et al. with a correction factor $f_{\mathrm{WR}}$ and with a metallicity dependence of $\dot{M} \propto\left(Z_{\text {init }} / Z_{\odot}\right)^{0.86}($ Vink \& de Koter 2005$)$ :

$$
\begin{aligned}
\log \left(\frac{\dot{M}_{\mathrm{WR}}}{M_{\odot} \mathrm{yr}^{-1}}\right)= & -11.95+1.5 \log L / L_{\odot}-2.85 X_{\mathrm{s}} \\
& +0.86 \log \left(Z_{\mathrm{init}} / Z_{\odot}\right)-\log f_{\mathrm{WR}} \\
& \text { for } \log L / L_{\odot}>4.5 \\
= & -35.8+6.8 \log L / L_{\odot}-2.85 X_{\mathrm{s}} \\
& +0.86 \log \left(Z_{\mathrm{init}} / Z_{\odot}\right)-\log f_{\mathrm{WR}} \\
& \text { for } \log L / L_{\odot} \leqslant 4.5 .
\end{aligned}
$$

We use $f_{\mathrm{WR}}=5$ or 10 in most model sequences, which means the WR mass-loss rate by Hamman et al. is lowered 5 or 10 times, to consider the most recent estimates (see Figure 1). The enhancement of stellar winds mass loss due to the centrifugal force is considered by

$$
\frac{\dot{M}}{\dot{M}\left(v_{\text {rot }}=0\right)}=\min \left[\left(\frac{1}{1-\Omega}\right)^{0.43}, 0.5 \frac{M}{\tau_{\mathrm{KH}}}\right] \text {, }
$$

where $\Omega=v_{\text {rot }} / v_{\text {crit }}$ with $v_{\text {crit }}=\sqrt{G M(1-\Gamma) / R}$ and $\Gamma$ is the Eddington factor (Langer 1998). Here, to prevent a singularity that may occur as $v_{\text {rot }}$ approaches $v_{\text {crit }}$, the mass-loss rate is limited to $\dot{M} \leqslant 0.5 M / \tau_{\mathrm{KH}}$, where $\tau_{\mathrm{KH}}$ is the thermal timescale of the star.

The binary orbit is assumed to be circular, and the Roche lobe radius is determined according to the approximation of Eggleton (1983). The mass-loss rate of the Roche lobe filling component through the first Lagrangian point is implicitly computed using the method given by Ritter (1988). The equation of motion of a test particle is numerically solved to calculate the amount of angular momentum of the accreted matter if the transferred matter directly hits the secondary star, and the Keplerian value is assumed otherwise (Wellstein 2001). The change of the orbital period due to mass transfer and stellar wind mass loss is considered according to Podsiadlowski et al. (1992). We follow Brookshaw \& Tavani (1993) to determine the amount of the specific angular momentum carried away from the orbit by stellar winds.

Tidal synchronization is considered following Wellstein (2001) (see also Detmers et al. 2008). We assume a synchronization timescale according to Tassoul $(1987,2000)$ who considered tidally driven meridional circulations as the main mechanism for tidal dissipation:

$$
\begin{aligned}
\tau_{\text {sync }}(\mathrm{yr})= & f_{\text {sync }} \frac{1.44 \times 10^{1.6}}{q(1+q)^{3 / 8}}\left(\frac{L_{\odot}}{L}\right)^{1 / 4} \\
& \times\left(\frac{M_{\odot}}{M}\right)^{1 / 8}\left(\frac{R}{R_{\odot}}\right)^{9 / 8}\left(\frac{d}{R}\right)^{33 / 8},
\end{aligned}
$$

where $q$ denotes the mass ratio and $d$ the orbital separation. This prescription gives a much shorter timescale than that given by Zahn (1977). Given that the physics of tidal dissipation is much debated in the literature (Langer 2009), we introduce a parameter $f_{\text {sync }}$ to investigate how an extremely fast/slow synchronization may influence the results. In most cases, however, we use $f_{\text {sync }}=1$.

A few sequences are also computed with $\tau_{\text {sync }}$ of Zahn (1977) for comparison:

$$
\begin{aligned}
\frac{1}{\tau_{\text {sync }}}= & 5\left(\frac{G M}{R^{3}}\right)^{1 / 2} q^{2}(1+q)^{5 / 6} \\
& \times \frac{M R^{2}}{I} E_{2}\left(\frac{R}{d}\right)^{17 / 2},
\end{aligned}
$$


where $I$ is the moment of the star, and $E_{2}$ a constant measuring the coupling between the tidal potential and the gravity mode. Using the data of Table 1 in Zahn (1977), we constructed a fitting formula for $E_{2}$ as the following:

$$
E_{2}=10^{-1.37}\left(\frac{R_{\text {conv }}}{R}\right)^{8},
$$

where $R_{\text {conv }}$ is the radius of the convective core. Note that both prescriptions by Tassoul and Zahn are not appropriate for a star with a convective envelope. ${ }^{5}$ However, the role of tidal synchronization is significant only on the main sequence and not important in late evolutionary stages as discussed below.

We computed 45 model sequences for initial masses of the primary star mostly from 12 to $25 M_{\odot}$ at two different metallicities ( $Z=0.02$ and 0.004 ), for different mass ratios, initial orbital periods, and WR mass-loss rates, as summarized in Table 1 . The initial rotational velocity at the equatorial surface of each star is set to be $20 \%$ of the Keplerian value. We could not calculate more massive systems because of a numerical difficulty encountered during the mass transfer phases, except for Seq. 26 where a primary star of $60 M_{\odot}$ is considered with a rather large WR mass-loss rate (i.e., $f_{\mathrm{WR}}=3$ ). The adopted initial orbital periods corresponds either to Case A or to Case B mass transfer. In the present study, we do not consider Case $\mathrm{C}$ systems, but briefly discuss the possible outcomes of Case $\mathrm{C}$ mass transfer in Section 6.4. The evolution of the primary stars is followed up to neon burning in most cases.

We also present non-rotating single helium star models to discuss SNe Ibc progenitors in binary systems with initial masses larger than $25 M_{\odot}$, and also to compare them with binary star models (Section 5).

\section{REDISTRIBUTION OF ANGULAR MOMENTUM IN PRIMARY STARS}

In this section, we focus our discussion on the evolution of primary stars and investigate whether binary evolution via Case A or Case B mass transfer could lead to diverse precollapse conditions of SNe Ibc in terms of the amount of core angular momentum. Although the evolution of mass-accreting secondary stars is a matter of extreme interest as discussed in Braun \& Langer (1995), Petrovic et al. (2005b) and Cantiello et al. (2007), it is beyond the scope of this paper.

Here, we first present some results including the Spruit-Tayler dynamo with our fiducial assumption on synchronization time (i.e., $f_{\text {sync }}=1$ ), showing that the final amount of angular momentum in the core of the primary star is not much affected by different histories of mass loss (i.e., Case AB or Case B; Section 4.1). Then, we discuss the influences of different assumptions on tidal synchronization and transport process of angular momentum (Section 4.2).

\subsection{Fiducial Models}

\subsubsection{Evolution with Case A and AB Mass Transfers}

The evolution of the primary star in a close binary system is characterized by the rapid loss of mass due to Roche lobe overflow. As an example, the evolution of the primary star in Seq. 14 is described in Figures 2 and 3, where our fiducial

\footnotetext{
5 On the other hand, note that a recent study by Toledano et al. (2007) suggests that intermediate-mass main-sequence stars follow the Zahn's synchronization timescale for convective stars.
}

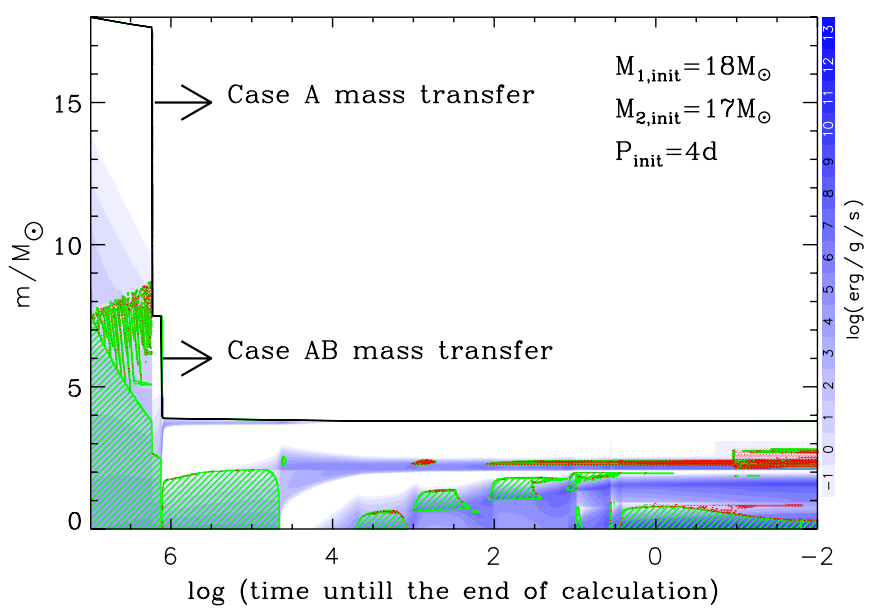

Figure 2. Evolution of the internal structure of the primary star in Seq. 14 $\left(M_{1, \text { init }}=18 M_{\odot}, M_{2, \text { init }}=17 M_{\odot}\right.$ and $P_{\text {init }}=4$ day $)$ from ZAMS to the neon burning phase. The hatched lines and the red dots denote convective layers and semi-convective layers, respectively. The different shades give the nuclear energy generation rate, for which the scale is shown on the right-hand side. The surface of the star is marked by the topmost solid line.

(A color version of this figure is available in the online journal.)

value of $f_{\text {sync }}=1$ is adopted, including the Spruit-Tayler dynamo. The binary system initially consists of a $18 M_{\odot}$ star and a $17 M_{\odot}$ star in a 4 day orbit. Mass transfer starts at $t=8.09 \times 10^{6} \mathrm{yr}$, when the helium mass fraction in the hydrogen burning core has increased to 0.94 . The mass transfer rate rises up to $8 \times 10^{-4} M_{\odot} \mathrm{yr}^{-1}$, which roughly corresponds to $M_{1} / \tau_{\mathrm{KH}, 1}$ where $M_{1}$ and $\tau_{\mathrm{KH}, 1}$ denote the mass and the Kelvin-Helmoltz timescale of the primary star, respectively. The primary mass decreases to $7.5 M_{\odot}$ by the end of the Case A transfer (see Figure 4). The second Roche lobe overflow begins at $t=8.513 \times 10^{6} \mathrm{yr}$ when the envelope of the primary star expands due to hydrogen shell burning during the helium core contraction phase (Case AB mass transfer). The primary star loses most of the hydrogen envelope as a result, exposing its helium core of $3.95 M_{\odot}$ having a small amount of hydrogen $\left(M_{\mathrm{H}}=0.04 M_{\odot}\right)$ in the outermost layers, as shown in the third panel of Figure 4.

Although the star remains compact $\left(R<0.9 R_{\odot}\right)$ during core helium burning, helium shell burning activated after core helium exhaustion leads to the expansion of the envelope up to $\sim 12 R_{\odot}$ (see Figure 3 ) during core carbon burning. A Case ABB mass transfer does not occur, however, due to the large orbital separation $\left(A=\sim 121 R_{\odot}\right)$ at this stage, while it does occur in many other sequences. The final mass at the end of the calculation (neon burning) is $3.79 M_{\odot}$. The mass of hydrogen decreases to $0.0015 M_{\odot}$ at the end, and the remaining mass of helium is $1.49 M_{\odot}$, as shown in the last panel of Figure 4 . The star is likely to eventually explode as a Type Ib supernova given the rather thick helium envelope with a very thin hydrogen layer, but it might also appear as Type IIb if the supernova were found within several days after the explosion (see Section 5.3).

The lower panel of Figure 3 shows that the mass transfer is not conservative. When the secondary star reaches critical rotation as a result of the accretion of angular momentum, the stellar wind mass-loss rate increases so drastically as to prevent efficient mass accumulation (see Petrovic et al. 2005a, for a more detailed discussion on this effect). The ratio of the accreted mass in the secondary star to the transferred mass from the primary star is about 0.83 during the Case A mass transfer, and 0.41 during 
Table 1

Properties of the Computed Sequences

\begin{tabular}{|c|c|c|c|c|c|c|c|c|c|c|c|c|c|}
\hline No. & $Z$ & $M_{1, \mathrm{i}}$ & $M_{2, \mathrm{i}}$ & $P_{\mathrm{i}}$ & $f_{\mathrm{WR}}$ & Case & $P_{\mathrm{f}}$ & $M_{1, \mathrm{f}}$ & $M_{\mathrm{CO}, \mathrm{f}}$ & $M_{\mathrm{He}}$ & $M_{\mathrm{H}}$ & $\left\langle j_{1.4}\right\rangle$ & Fate \\
\hline 1 & 0.02 & 12 & 8 & 3.0 & 5 & $\mathrm{~B}+\mathrm{BB}$ & 57.9 & $1.40^{\mathrm{a}}$ & $1.21^{\mathrm{a}}$ & $0.17^{\mathrm{a}}$ & 0.0 & 0.33 & ONeMg WD \\
\hline 2 & 0.02 & 12 & 11 & 4.0 & 5 & $\mathrm{~B}+\mathrm{BB}$ & 104.4 & $1.48^{\mathrm{a}}$ & $1.24^{\mathrm{a}}$ & $0.20^{\mathrm{a}}$ & 0.0 & 0.35 & ONeMg WD \\
\hline 3 & 0.02 & 13 & 11 & 5.0 & 5 & $\mathrm{~B}+\mathrm{BB}$ & 123.3 & $1.64^{\mathrm{a}}$ & $1.43^{\mathrm{a}}$ & $0.18^{\mathrm{a}}$ & 0.0 & 0.22 & SN Ic \\
\hline 4 & 0.02 & 14 & 12 & 3.0 & 5 & $\mathrm{~A}+\mathrm{AB}+\mathrm{BB}$ & 118.5 & $1.33^{\mathrm{a}}$ & $1.09^{\mathrm{a}}$ & $0.22^{\mathrm{a}}$ & 0.0 & $\ldots$ & ONeMg WD \\
\hline 5 & 0.02 & 14 & 12 & 5.0 & 5 & $\mathrm{~B}+\mathrm{BB}$ & 30.7 & 2.97 & 1.66 & 1.24 & $1.9(-4)$ & 0.25 & SNIb \\
\hline 6 & 0.02 & 16 & 14 & 2.0 & 5 & A:Contact & & & & & & & \\
\hline 7 & 0.02 & 16 & 14 & 3.0 & 5 & $\mathrm{~A}+\mathrm{AB}+\mathrm{ABB}$ & 101.8 & $1.54^{\mathrm{a}}$ & $1.33^{\mathrm{a}}$ & $0.17^{\mathrm{a}}$ & 0.0 & 0.39 & ONeMg WD \\
\hline 8 & 0.02 & 16 & 14 & 4.0 & 5 & $\mathrm{~B}+\mathrm{BB}$ & 26.2 & 3.66 & 2.05 & 1.47 & $4.5(-3)$ & 0.24 & SNIb \\
\hline 9 & 0.02 & 16 & 14 & 5.0 & 5 & $\mathrm{~B}+\mathrm{BB}$ & 33.7 & 3.65 & 2.04 & 1.47 & $5.0(-3)$ & 0.25 & SNIb \\
\hline 10 & 0.02 & 18 & 12 & 3.0 & 5 & $\mathrm{~A}+\mathrm{AB}+\mathrm{ABB}$ & 27.9 & 2.66 & 1.58 & 1.01 & 0.00 & 0.26 & $\mathrm{SNIb}$ \\
\hline 11 & 0.02 & 18 & 12 & 3.0 & 10 & $\mathrm{~A}+\mathrm{AB}+\mathrm{ABB}$ & 27.3 & 2.74 & 1.59 & 1.08 & 0.00 & 0.26 & SNIb \\
\hline 12 & 0.02 & 18 & 12 & 5.0 & 10 & B:Contact & & & & & & & \\
\hline 13 & 0.02 & 18 & 17 & 3.0 & 10 & $\mathrm{~A}+\mathrm{AB}+\mathrm{ABB}$ & 36.2 & 3.03 & 1.68 & 1.27 & $7.9(-4)$ & 0.25 & $\mathrm{SN} \mathrm{Ib}$ \\
\hline 14 & 0.02 & 18 & 17 & 4.0 & 10 & $\mathrm{~A}+\mathrm{AB}$ & 29.7 & 3.79 & 2.14 & 1.49 & $1.5(-3)$ & 0.25 & $\mathrm{SN} \mathrm{Ib}$ \\
\hline $15^{\mathrm{b}}$ & 0.02 & 18 & 17 & 4.0 & 10 & $\mathrm{~A}+\mathrm{AB}$ & 24.0 & 3.97 & 2.27 & 1.53 & $1.0(-3)$ & 0.26 & SNIb \\
\hline $16^{\mathrm{c}}$ & 0.02 & 18 & 17 & 4.0 & 10 & $\mathrm{~A}+\mathrm{AB}$ & 50.0 & 3.80 & 2.16 & 1.50 & $2.2(-3)$ & 0.06 & SNIb \\
\hline $17^{\mathrm{d}}$ & 0.02 & 18 & 17 & 4.0 & 10 & $\mathrm{~A}+\mathrm{AB}$ & 25.2 & 3.84 & 2.18 & 1.50 & $1.4(-3)$ & 0.26 & $\mathrm{SNIb}$ \\
\hline $18^{\mathrm{e}}$ & 0.02 & 18 & 17 & 4.0 & 10 & $A+A B$ & 30.6 & 3.73 & 2.14 & 1.43 & 0.00 & 3.57 & $\mathrm{SNIb}$ \\
\hline 19 & 0.02 & 18 & 17 & 5.0 & 3 & B & 33.1 & 3.73 & 2.33 & 1.23 & 0.00 & 0.25 & $\mathrm{SNIb}$ \\
\hline 20 & 0.02 & 18 & 17 & 5.0 & 5 & B & 32.4 & 4.04 & 2.45 & 1.4 & 0.00 & 0.33 & $\mathrm{SNIb}$ \\
\hline 21 & 0.02 & 18 & 17 & 5.0 & 10 & $\mathrm{~B}+\mathrm{BB}$ & 31.5 & 4.41 & 2.51 & 1.68 & $9.9(-3)$ & 0.26 & $\mathrm{SNIb}$ \\
\hline 22 & 0.02 & 18 & 17 & 6.0 & 10 & B & 39.3 & 4.39 & 2.56 & 1.62 & $4.0(-3)$ & 0.26 & $\mathrm{SNIb}$ \\
\hline 23 & 0.02 & 25 & 19 & 6.0 & 10 & B:Contact & & & & & & & \\
\hline 24 & 0.02 & 25 & 24 & 2.0 & 10 & A:Contact & & & & & & & \\
\hline 25 & 0.02 & 25 & 24 & 3.0 & 3 & $\mathrm{~A}+\mathrm{AB}$ & 22.7 & 3.70 & 2.46 & 0.98 & 0.0 & 0.24 & $\mathrm{SNIb}$ \\
\hline 26 & 0.02 & 25 & 24 & 3.0 & 5 & $\mathrm{~A}+\mathrm{AB}$ & 22.4 & 4.33 & 2.80 & 1.30 & 0.0 & 0.25 & SNIb \\
\hline 27 & 0.02 & 25 & 24 & 3.0 & 10 & $\mathrm{~A}+\mathrm{AB}$ & 21.3 & 5.07 & 3.17 & 1.67 & 0.0 & 0.25 & SNIb \\
\hline $28^{\mathrm{d}}$ & 0.02 & 25 & 24 & 3.0 & 10 & $\mathrm{~A}+\mathrm{AB}$ & 18.9 & 5.08 & 3.19 & 1.66 & 0.0 & 0.32 & $\mathrm{SNIb}$ \\
\hline 29 & 0.02 & 25 & 24 & 4.0 & 5 & $\mathrm{~A}+\mathrm{AB}$ & 21.5 & 4.45 & 2.91 & 1.22 & 0.0 & 0.26 & $\mathrm{SNIb}$ \\
\hline 30 & 0.02 & 25 & 24 & 6.0 & 10 & B & 27.4 & 6.49 & 4.45 & 1.63 & 0.0 & 0.39 & SNIb \\
\hline 31 & 0.02 & 60 & 40 & 7.0 & 3 & A & 16.8 & 4.95 & 3.70 & 0.25 & 0 & 0.24 & SNIc \\
\hline 32 & 0.004 & 16 & 12 & 3.0 & 5 & $\mathrm{~B}+\mathrm{BB}$ & 64.75 & 3.91 & 2.22 & 1.54 & $1.6(-2)$ & 0.24 & SNIb \\
\hline 33 & 0.004 & 16 & 14 & 3.0 & 5 & $\mathrm{~B}+\mathrm{BB}$ & 19.6 & 3.90 & 2.21 & 1.53 & $1.6(-2)$ & 0.24 & $\mathrm{SNIb}$ \\
\hline 34 & 0.004 & 16 & 14 & 5.0 & 5 & $\mathrm{~B}+\mathrm{BB}$ & 21.8 & 3.84 & 2.19 & 1.51 & $1.2(-2)$ & 0.24 & $\mathrm{SNIb}$ \\
\hline 35 & 0.004 & 18 & 12 & 5.0 & 5 & $\mathrm{~B}+\mathrm{BB}$ & 14.4 & 4.64 & 2.76 & 1.68 & $1.7(-2)$ & 0.31 & $\mathrm{SNIb}$ \\
\hline 36 & 0.004 & 18 & 12 & 8.0 & 5 & $\mathrm{~B}+\mathrm{BB}$ & 24.33 & 4.56 & 2.68 & 1.67 & $1.5(-2)$ & 0.33 & $\mathrm{SNIb}$ \\
\hline 37 & 0.004 & 18 & 17 & 3.0 & 5 & $\mathrm{~A}+\mathrm{AB}$ & 18.4 & 4.42 & 2.55 & 1.67 & $2.6(-2)$ & 0.26 & $\mathrm{SNIb}$ \\
\hline 38 & 0.004 & 18 & 17 & 3.0 & 10 & $\mathrm{~A}+\mathrm{AB}$ & 14.4 & 4.58 & 2.61 & 1.77 & $2.7(-2)$ & 0.26 & SNIb \\
\hline 39 & 0.004 & 18 & 17 & 6.0 & 5 & $\mathrm{~B}+\mathrm{BB}$ & 27.0 & 4.57 & 2.71 & 1.65 & $1.6(-2)$ & 0.27 & $\mathrm{SNIb}$ \\
\hline 40 & 0.004 & 25 & 12 & 3.0 & 5 & A:Contact & & & & & & & \\
\hline 41 & 0.004 & 25 & 12 & 6.0 & 5 & B:Contact & & & & & & & \\
\hline 42 & 0.004 & 25 & 19 & 3.0 & 5 & $\mathrm{~A}+\mathrm{AB}$ & 10.2 & 7.09 & 4.87 & 2.03 & $6.5(-3)$ & 0.32 & SNIb \\
\hline 43 & 0.004 & 25 & 24 & 3.0 & 5 & $\mathrm{~A}+\mathrm{AB}$ & 13.0 & 7.31 & 5.05 & 2.07 & $8.7(-3)$ & 0.28 & $\mathrm{SNIb}$ \\
\hline 44 & 0.004 & 25 & 24 & 6.0 & 5 & B:Contact & & & & & & & \\
\hline 45 & 0.004 & 40 & 30 & 4.0 & 5 & $\mathrm{~A}+\mathrm{AB}$ & 9.31 & 12.0 & 9.42 & 1.24 & 0.0 & 0.56 & $\mathrm{BH}$ \\
\hline
\end{tabular}

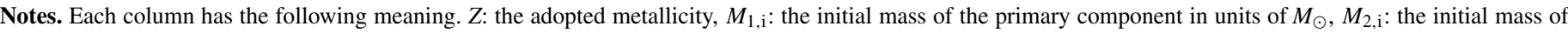

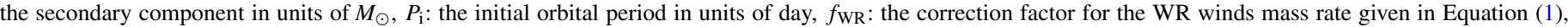



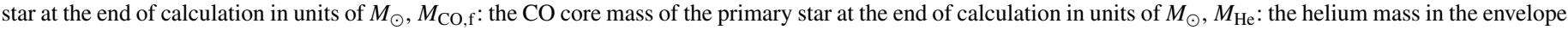

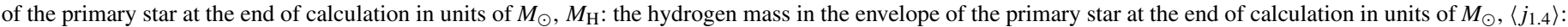

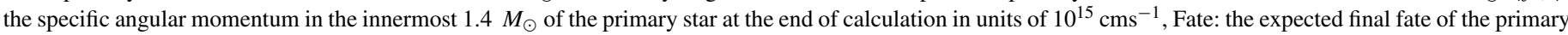
star.

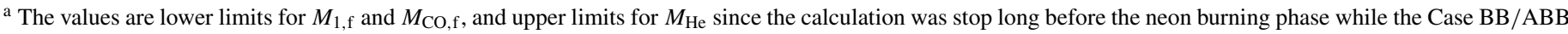
mass transfer phase was not finished.

b $f_{\text {sync }}=10^{5}$.

c $f_{\text {sync }}=0.01$.

d $\tau_{\text {sync }}$ according to Zahn.

e Non-magnetic model.

the Case B mass transfer. The previous calculations by WL99 and Wellstein et al. (2001) show that the non-conservative mass transfer leads to a shorter orbit than in the case of conservative mass transfer, in general. This effect should be kept in mind in the following discussion on the evolution of the binary orbit and its consequences.

The evolution of rotation in the primary star is shown in Figures 5, 6 and 7. As shown in Figure 5, the total angular mo- 

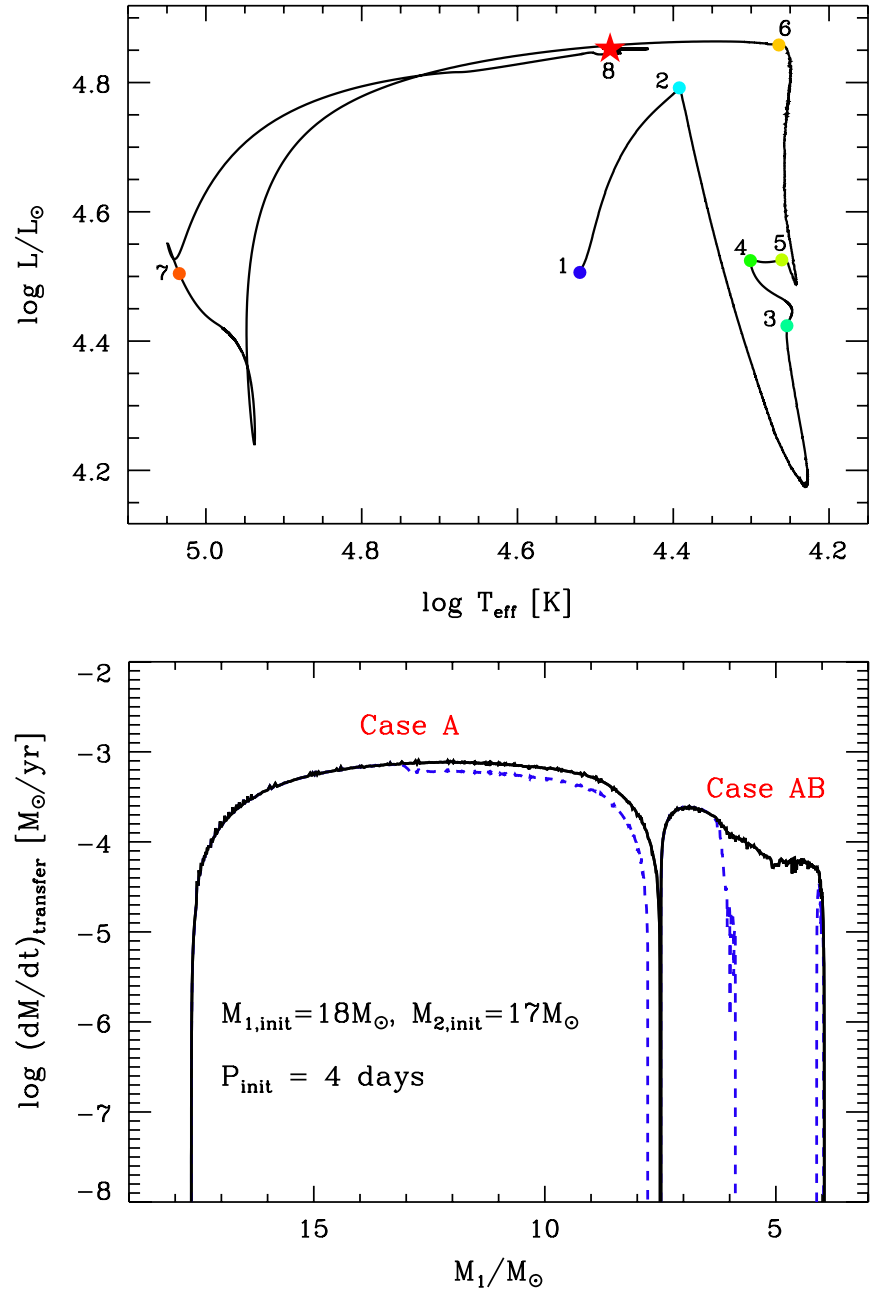

Figure 3. Upper panel: evolutionary track of the primary star in Seq. 14 $\left(M_{1 \text {,init }}=18 M_{\odot}, M_{2 \text {,init }}=17 M_{\odot}\right.$ and $P_{\text {init }}=4$ day $)$ in the HR diagram. The filled circles on the track mark different evolutionary epochs as the following. 1: ZAMS, 2: beginning of the Case A mass transfer, 3: end of the Case A mass transfer, 4: core hydrogen exhaustion, 5: beginning of the Case $\mathrm{AB}$ mass transfer, 6: end of the Case $\mathrm{AB}$ mass transfer, 7: core helium exhaustion, and 8: neon burning (end of calculation). Lower panel: mass transfer rates from the primary star (solid line) and mass accretion rates onto the secondary star (dashed line) during the Case $\mathrm{A}$ and $\mathrm{AB}$ transfers as a function of the primary star mass.

(A color version of this figure is available in the online journal.)

mentum of the primary star rapidly decreases in the beginning as a result of tidal interaction, until the star is completely synchronized with the orbit when $t \simeq 2 \times 10^{5}$ yr. Figure 6 shows the evolution of the angular velocity inside the primary star from ZAMS until it reaches complete synchronization. Note that the star keeps rotating almost rigidly, even though the tidal interaction causes redistribution of angular momentum from the outermost layers of the star (Wellstein 2001; Detmers et al. 2008). This results from the very short timescale of the transport of angular momentum in the star (about 300 years) due mainly to convection and the Spruit-Tayler dynamo, in the convective and radiative layers, respectively.

Later on, the angular velocity of the primary star still remains coupled with the orbital motion, until decoupling starts during Case B mass transfer (see below). Interestingly the total angular momentum of the primary star gradually increases from the initial synchronization until the onset of Case A mass transfer (Figure 5). This is because the star significantly expands, while the change of the orbital separation remains small during this period, as shown in the third and last panels in Figure 7. However, it rapidly decreases again during the Case $\mathrm{A}$ and $\mathrm{AB}$ mass transfer phases, as explained below.

Figure 7 shows that angular momentum in the core of the primary star is mostly removed during the mass transfer phases (Case A and Case AB). It should be noted, however, that the mechanism for braking the core is different for each case. During the Case A mass transfer, the synchronization timescale according to Equation (3) remains very short $\left(10^{3} \mathrm{yr}\right)$ compared to the mass transfer timescale $\left(10^{4} \mathrm{yr}\right)$. The decrease of the core angular momentum during the Case A mass transfer results from the synchronization that occurs even when the orbit is rapidly widened due to mass exchange (Figure 7). During the Case $A B$ mass transfer, the further increase of the orbital separation significantly weakens the role of synchronization for the redistribution of angular momentum. However, both the Spruit-Tayler dynamo and mass loss lead to rapid braking of the thermally contracting helium core. Further significant corebraking by the Spruit-Tayler dynamo occurs during the CO core contraction phase, and the mean specific angular momentum of the innermost $1.4 M_{\odot}$ becomes about $2.5 \times 10^{14} \mathrm{~cm}^{2} \mathrm{~s}^{-1}$ at the neon burning phase.

\subsubsection{Evolution with Case B Mass Transfer}

In Seq. 20, the initial masses of the stellar components are the same as in Seq. 14 but the initial period is large enough that the first mass transfer occurs during the helium core contraction phase (Case B mass transfer). Avoiding Case A mass transfer, the primary star retains more angular momentum in the core at the end of main sequence than in Seq. 14. However, the core loses more angular momentum during the helium core contraction phase than in Seq. 14, as stronger magnetic torques are exerted, mainly due to the more massive envelope (Figure 8). At neon burning, $\left\langle j_{1.4}\right\rangle=2.6 \times 10^{14} \mathrm{~cm}^{2} \mathrm{~s}^{-1}$ is obtained, which is similar to that in Seq. 14.

\subsection{Non-fiducial Models}

\subsubsection{Influence of the Synchronization Timescale}

The effect of synchronization is negligible in Seq. 15, where $f_{\text {sync }}=10^{5}$ is adopted. As shown in Figure 9, a rather rapid decrease of $\left\langle j_{\text {core }}\right\rangle$ occurs during and after the Case A mass transfer, which is a combined effect of mass loss and magnetic torques: mass loss carries away angular momentum from the envelope, and magnetic torques brake the core rotation subsequently. The core is further slowed down during the helium core and $\mathrm{CO}$ core contraction phases, resulting in $\left\langle j_{1.4}\right\rangle=2.6 \times 10^{14} \mathrm{~cm}^{2} \mathrm{~s}^{-1}$ at the neon burning phase. Note that this value is very close to that in Seq. 14 .

We find that the result with Zahn's prescription for synchronization (Seq. 17) is not much different from that of Seq. 15 where synchronization is negligible. The synchronization timescale according to Zahn is sensitive to the ratio of the convective core size to the stellar radius $\left(1 / \tau_{\mathrm{sync}} \propto\left(R_{\text {conv }} / R_{\mathrm{star}}\right)^{8}\right.$, see Equations (4) and (5)). This ratio continuously decreases as the star evolves, and thus $\tau_{\text {sync }}$ continuously increases to such an extent that the effect of synchronization can be ignored when Case A mass transfer starts. When $f_{\text {sync }}=0.01$ with Tassoul's prescription is used (Seq. 16), on the other hand, synchronization becomes important even after the Case $\mathrm{AB}$ mass transfer phase, and the primary is further spun down by tidal interaction, giving $\left\langle j_{1.4}\right\rangle=610^{13} \times \mathrm{cm}^{2} \mathrm{~s}^{-1}$ at the neon burning phase. 

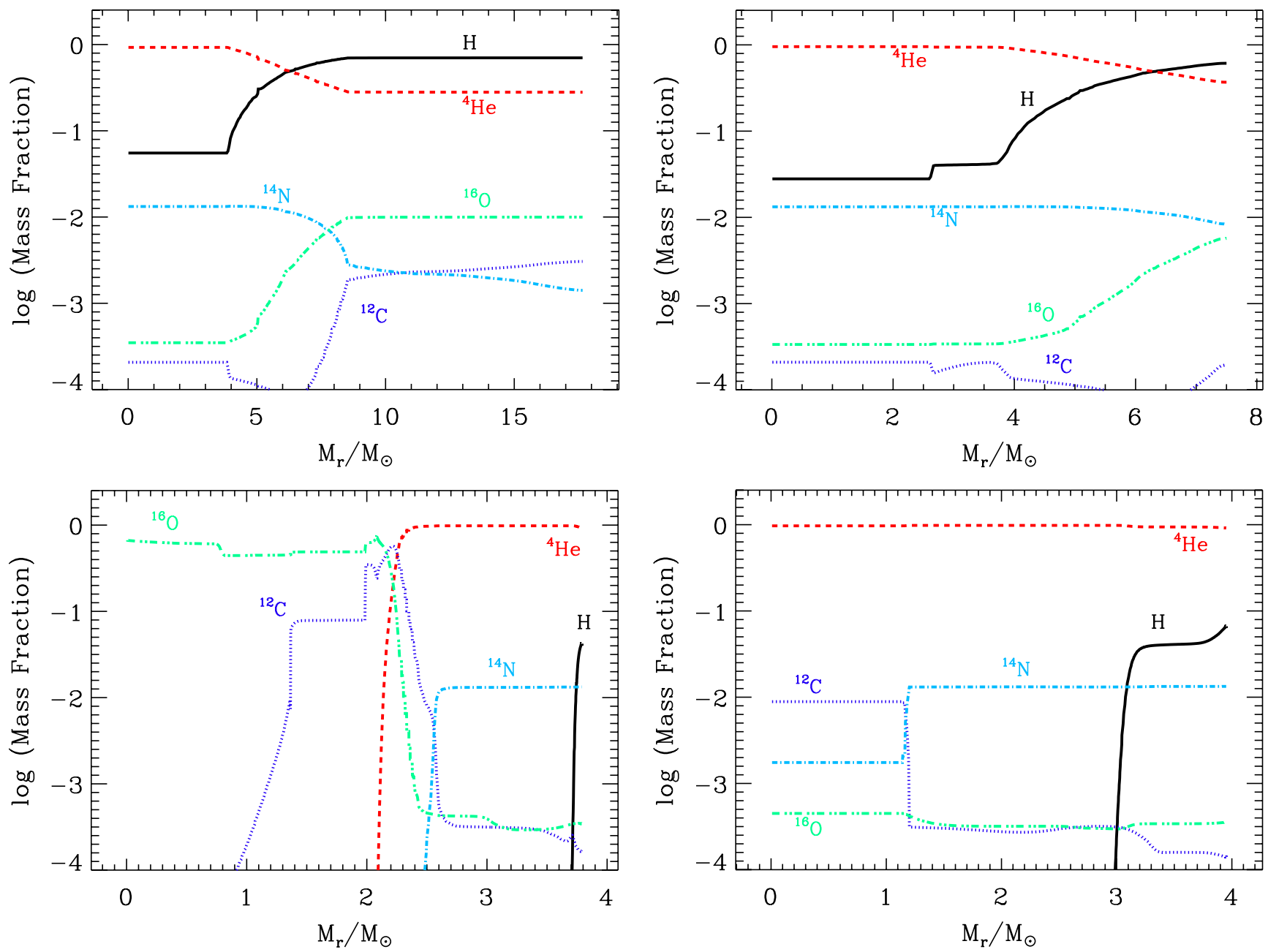

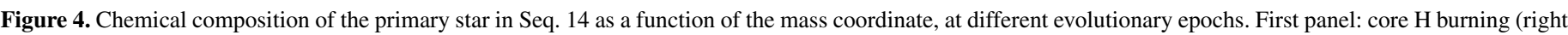

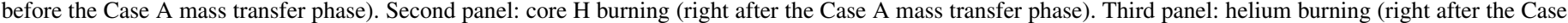
AB mass transfer phase). Last panel: neon burning.

(A color version of this figure is available in the online journal.)

\subsubsection{Non-magnetic Model}

In Seq. 18 where the Spruit-Tayler dynamo is not included, the core is spun down due to synchronization during the Case A mass transfer phase (Figure 9) as in the corresponding magnetic case (Seq. 14; Figure 7). The spin-orbit coupling becomes significantly weakened as the orbit widens after the Case A mass transfer phase. Despite a significant amount of mass being lost via Case $\mathrm{AB}$ mass transfer, the core in the primary star retains most of the remaining angular momentum in the following evolutionary stages. This is because the chemical gradient across the boundary between the helium core and the hydrogen envelope effectively prohibits the transport of angular momentum (cf. Meynet \& Maeder 1997; Heger et al. 2000). The core angular momentum at neon burning is thus about 10 times larger $\left(\left\langle j_{1.4}\right\rangle=3.5710^{15} \mathrm{~cm}^{2} \mathrm{~s}^{-1}\right)$ than in the corresponding magnetic case.

\subsection{Discussion}

As shown in the above examples, in the model sequences with the Spruit-Tayler dynamo, all of the primary stars retain similar amounts of angular momentum (a few $10^{14} \mathrm{~cm}^{2} \mathrm{~s}^{-1}$ ) in the innermost $1.4 M_{\odot}$ at neon burning regardless of the detailed history of mass transfer, unless synchronization is extremely fast as in Seq. 16 (see Table 1). According to the Spruit-Tayler dynamo, magnetic torques exerted to the core become stronger with a higher spin rate, a larger degree of differential rotation between the core and the envelope, and a heavier radiative envelope. Therefore, although winds or Roche lobe overflows reduce the size of the hydrogen envelope and remove angular momentum from the star, this in turn weakens the torque exerted to the core, and vice versa. The remarkable convergence of $\left\langle j_{1.4}\right\rangle$ to a few $10^{14} \mathrm{~cm}^{2} \mathrm{~s}^{-1}$ in our model sequences, even for different wind parameters and metallicities as shown in Table 1, can be explained by this self-regulating nature of the Spruit-Tayler dynamo. This result indicates that not much diversity is expected in $\mathrm{SNe}$ Ibc progenitors produced via Case $\mathrm{A}$ or Case $\mathrm{AB} / \mathrm{B}$ mass transfer, in terms of rotation: most $\mathrm{SNe}$ Ibc of a similar progenitor mass may leave neutron stars with a similar spin rate. However, other types of binary interactions still may lead to various final rotation periods in SN progenitors (see Brown et al. 2000; Cantiello et al. 2007; Podsiadlowski et al. 2010, for such examples).

\section{THE NATURE OF SN Ibc PROGENITORS}

In the literature, the detailed history of binary interactions such as mass transfer and tidal interaction is often neglected, and only the evolution of pure helium stars to discuss SNe Ibc 


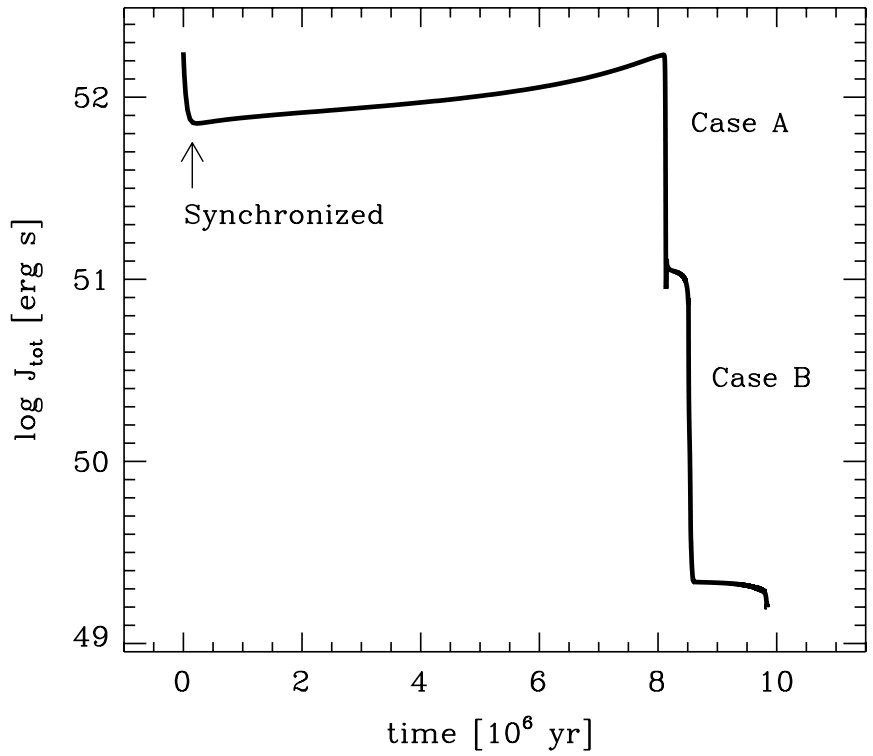

Figure 5. Evolution of the total angular momentum of the primary star in Seq. 14 , as a function of time, from the ZAMS until neon burning.

progenitors in binary systems is investigated (e.g., WLW95; Pols \& Dewi 2002). Although this approach is very useful, our binary star models indicate that complications resulting from binary interactions have important consequences in SN Ibc progenitors. To address this issue, here we also present evolutionary models of non-rotating single helium stars with initial masses of 2.8-20 $M_{\odot}$ at $Z=0.02$ for comparison with binary star models, as presented in Table 2. This also enables us to study SN Ibc progenitors with initial masses higher than $25 M_{\odot}$, which are lacking in our binary model sequences. These helium star models were calculated up to neon burning, with the WR mass-loss rate given in Equation (1) with $f_{\mathrm{WR}}=5$ or 10 , as in the binary models. In the following, we focus our discussion mostly on the results with $f_{\mathrm{WR}}=5$, while the influence of the wind parameter is briefly discussed.

\subsection{Final Mass}

The final mass of the primary stars in close binary systems is largely determined by the winds mass loss during core helium burning, if the initial mass $\left(M_{\mathrm{He}, \mathrm{i}}\right.$, i.e., the mass right after Case $\mathrm{AB}$ or Case B mass transfer) is significantly larger than about 3.0 $M_{\odot}$. However, for a less massive helium star, the expansion of the helium envelope becomes so dramatic during $\mathrm{CO}$ core contraction, and/or during core carbon burning, that it can lead to another mass transfer phase: Case ABB or Case BB (e.g., compare the final radius of the $2.8 M_{\odot}$ helium star model with those of more massive helium star models in Table 2).

The impact of this mass transfer phase becomes more important for a less massive helium star. In Seqs. 1, 2, and 4 where $M_{\mathrm{He}, \mathrm{i}} \simeq 2.1-2.3 M_{\odot}$, the rapid loss of mass during Case $\mathrm{ABB} / \mathrm{BB}$ transfer reduces the total mass of the primary stars to such an extent that they may not explode as supernovae, but die as white dwarfs. In Seq. 3 , where $M_{\mathrm{He}, \mathrm{i}} \simeq 2.7 M_{\odot}$, the carbon-oxygen core can grow beyond the Chandrasekhar limit despite the significant loss of mass (i.e., about $1 M_{\odot}$ ) during the Case BB phase. The remaining helium mass in the envelope is only about $0.18 M_{\odot}$ at core carbon exhaustion. For the other sequences at solar metallicity (Seqs. 5, 8, 9, 10, 11, 13, and 21) where $M_{\mathrm{He}, \mathrm{i}}>3.0 M_{\odot}$, the amounts of mass loss via Case

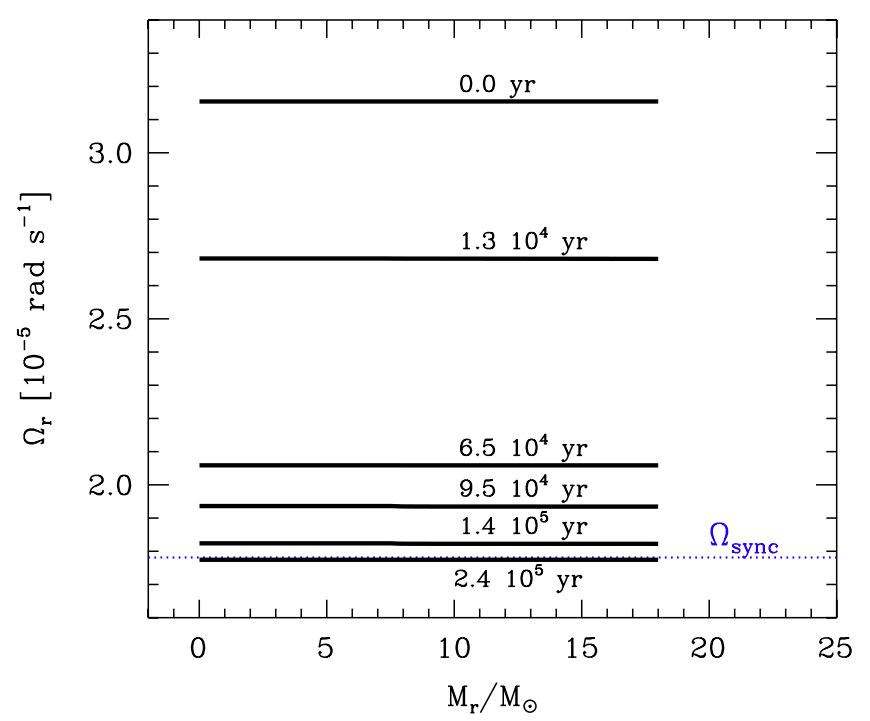

Figure 6. Angular velocity profile in the primary star of Seq. 14 as a function of the mass coordinate, at six different epochs from the ZAMS until $2.4 \times 10^{5}$ yr. The thin dotted line denotes the angular velocity of the orbit (i.e., $\left.\Omega_{\text {sync }}:=2 \pi / P_{\text {orbit }}\right)$ at $2.4 \times 10^{5} \mathrm{yr}$.

(A color version of this figure is available in the online journal.)

Table 2

Properties of the Computed Single Helium Stars at $Z=0.02$

\begin{tabular}{lrcrrrrc}
\hline \hline$f_{\mathrm{WR}}$ & $M_{\mathrm{He}, \mathrm{i}}$ & $M_{\mathrm{f}}$ & $M_{\mathrm{CO}, \mathrm{f}}$ & $M_{\mathrm{He}}$ & \multicolumn{1}{c}{$R$} & $R_{95}$ & $E_{b}$ \\
\hline 5 & 2.8 & 2.73 & 1.51 & 1.16 & 163.54 & 5.08 & 0.01 \\
5 & 3.0 & 2.91 & 1.60 & 1.22 & 33.40 & 1.79 & 0.03 \\
5 & 4.0 & 3.72 & 2.15 & 1.43 & 8.01 & 0.73 & 0.18 \\
5 & 6.0 & 4.23 & 2.77 & 1.19 & 4.19 & 0.38 & 0.25 \\
5 & 8.0 & 4.95 & 3.40 & 0.85 & 3.07 & 0.30 & 0.29 \\
5 & 10.0 & 5.49 & 3.93 & 0.54 & 1.59 & 0.22 & 0.47 \\
5 & 15.0 & 6.44 & 4.85 & 0.31 & 0.59 & 0.18 & 0.55 \\
5 & 20.0 & 7.09 & 5.44 & 0.22 & 0.48 & 0.16 & 0.66 \\
\hline 10 & 3.0 & 2.95 & 1.60 & 1.27 & 34.96 & 1.84 & 0.03 \\
10 & 4.0 & 3.84 & 2.20 & 1.49 & 6.94 & 0.70 & 0.15 \\
10 & 6.0 & 4.93 & 3.14 & 1.55 & 4.29 & 0.51 & 0.19 \\
10 & 8.0 & 6.06 & 4.13 & 1.41 & 2.55 & 0.35 & 0.33 \\
10 & 10.0 & 7.01 & 4.96 & 1.12 & 1.94 & 0.25 & 0.55 \\
10 & 15.0 & 8.89 & 6.80 & 0.47 & 0.54 & 0.20 & 0.82 \\
10 & 20.0 & 10.33 & 8.21 & 0.31 & 0.40 & 0.16 & 1.17 \\
\hline
\end{tabular}

Notes. Each column has the following meaning. $f_{\mathrm{WR}}$ : the correction factor for the WR winds mass rate given in Equation (1), $M_{\mathrm{He}, \mathrm{i}}$ : the initial mass of the helium star in units of $M_{\odot}, M_{\mathrm{f}}$ : the final mass at the end of calculation (i.e., at neon burning) in units of $M_{\odot}, M_{\mathrm{CO}, \mathrm{f}}$ : the $\mathrm{CO}$ core mass at the end of calculation in units of $M_{\odot}, M_{\mathrm{He}}$ : the amount of helium in the envelope at the end of calculation in units of $M_{\odot}, R$ : the radius at the end of calculation in units of $R_{\odot}, R_{95}$ : the radius that encompasses $95 \%$ of the total mass at the end of calculation in units of $R_{\odot}, E_{\mathrm{b}}$ : the binding energy of the envelope above $1.5 M_{\odot}$ at the end of calculation in units of $10^{51} \mathrm{erg}$.

$\mathrm{ABB} / \mathrm{BB}$ transfer until core neon burning are rather moderate, varying from 0.02 to $0.1 M_{\odot}$.

The WR wind mass-loss rate has significant consequences in systems where $M_{\mathrm{He}, \mathrm{i}}>3.0 M_{\odot}$. The previous study by WLW95 concluded that the final masses of single helium stars with initial masses of 3-20 $M_{\odot}$ should converge to $\sim 2.2-3.5 M_{\odot}$. As expected from the lower WR mass-loss rate adopted in this study, our helium star models give a significantly wider range of final masses $\left(M_{\mathrm{f}}\right)$ at the given metallicity of $0.02: M_{\mathrm{f}}=2.9-7 M_{\odot}$ $\left(2.9-10 M_{\odot}\right)$ from helium stars of $3-20 M_{\odot}$ for $f_{\mathrm{WR}}=5$ $\left(f_{\mathrm{WR}}=10\right.$; Table 2 ; cf. Figure 14$)$. Our binary star models 

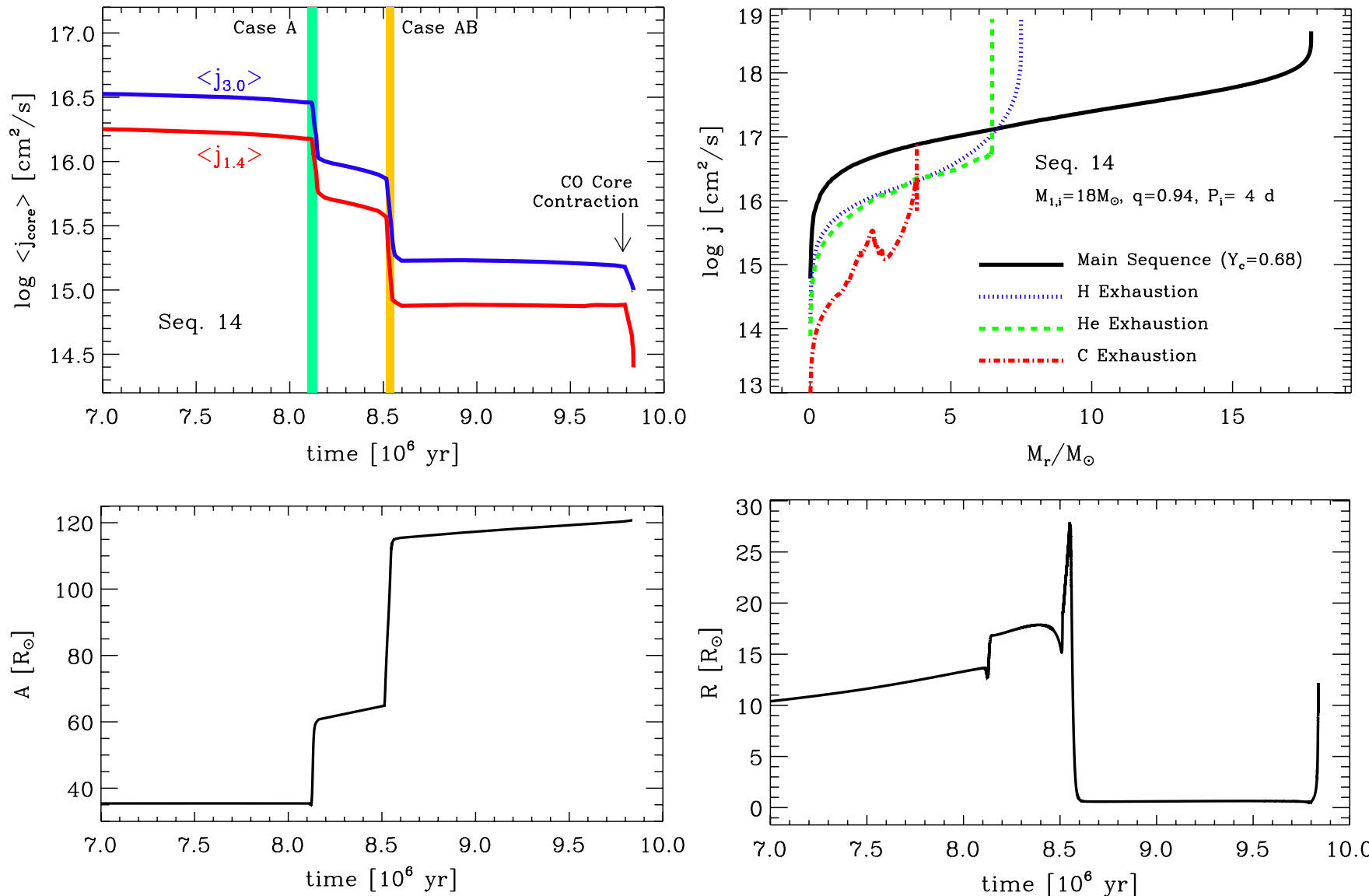

Figure 7. Top panel: mean specific angular momentum of the innermost $3 M_{\odot}$ and $1.4 M_{\odot}$ of the primary star in Seq. 14 as a function of the evolutionary time. The time spans for the Case A and $\mathrm{AB}$ mass transfers are marked by the color shades as indicated by the labels. Second panel: distribution of angular momentum in the primary star in Seq. 9 at different evolutionary stages, as indicated by the labels. Third panel: the orbital separation of the binary system in Seq. 14, as a function of time. Bottom panel: the radius change of the primary star in Seq. 14, as a function of time.

(A color version of this figure is available in the online journal.)

also give generally larger final masses for SN Ibc progenitors than those in WL99: $M_{\mathrm{f}} \simeq 1.64-4.5 M_{\odot}$ for $f_{\mathrm{WR}}=5$ from $M_{\mathrm{ZAMS}}=13-25 M_{\odot}$, compared to $M_{\mathrm{f}} \simeq 2.0-3.4 M_{\odot}$ in WL99. ${ }^{6}$

\subsection{Helium}

Figure 10 shows the amount of helium $\left(M_{\mathrm{He}}\right)$ as a function of the final mass in the SN Ibc progenitor models with $f_{\mathrm{WR}}=5$ at solar metallicity. In general, both binary and helium star models predict large amounts of helium in the envelope compared to the results of WLW95 and WL99 except for relatively lowmass systems of $M_{\mathrm{He}, \mathrm{i}} \lesssim 3.0 M_{\odot}$. Note that only rather massive progenitors with $M_{\mathrm{f}} \gtrsim 5.5 M_{\odot}$ can be significantly helium deficient (i.e., $M_{\mathrm{He}}<0.5 M_{\odot}$ ).

For the systems with $M_{\mathrm{f}} \lesssim 3.0 M_{\odot}$, the role of Case $\mathrm{ABB} / \mathrm{BB}$ mass transfer becomes important. In particular, the small amount of helium $\left(M_{\mathrm{He}} \lesssim 0.18 M_{\odot}\right)$ in Seq. 3 shows that helium deficiency can also be achieved by the so-called Case $\mathrm{BB} / \mathrm{ABB}$ mass transfer from relatively low-mass helium cores $\left(M_{\mathrm{He}, \mathrm{i}}<3 M_{\odot}\right)$. Therefore, we expect two different classes for Type Ic progenitors at solar metallicity: one with $M_{\mathrm{f}} \lesssim 2.0 M_{\odot}$ and the other with $M_{\mathrm{f}} \gtrsim 5.5 M_{\odot}$ (see also Wellstein \& Langer 1999; Pols \& Dewi 2002).

\footnotetext{
6 Here, the lower end of $M_{\mathrm{f}}$ is determined by the detailed history of Case $\mathrm{ABB} / \mathrm{BB}$ transfer, which depends on uncertain parameters such as the semi-convection efficiency.
}

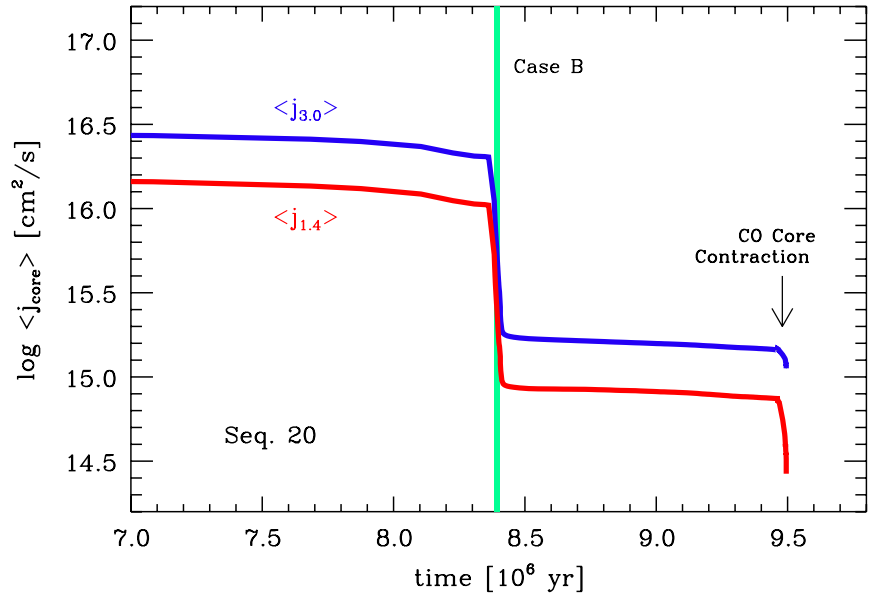

Figure 8. Mean specific angular momentum of the innermost $3 M_{\odot}$ and $1.4 M_{\odot}$ of the primary star in Seq. 20, as a function of the evolutionary time. The time spans for the Case B mass transfers are marked by the color shades as indicated by the labels.

(A color version of this figure is available in the online journal.)

Obviously, the upper limit of $M_{\mathrm{f}}$ for potential Type Ic progenitors increases with a smaller mass-loss rate: with $f_{\mathrm{WR}}=$ 10 , we have $M_{\mathrm{f}} \gtrsim 8.9 M_{\odot}$. 

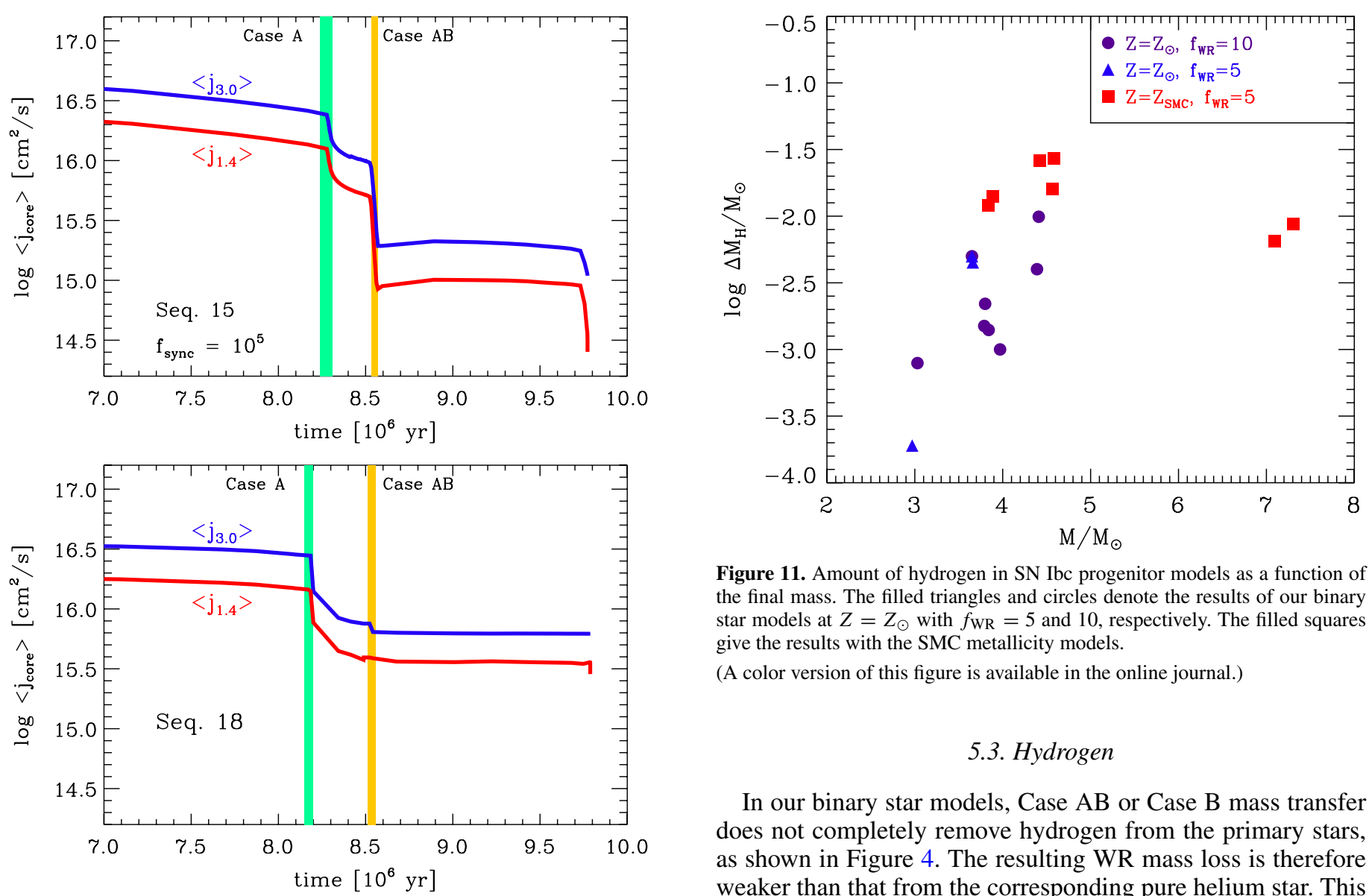

Figure 11. Amount of hydrogen in SN Ibc progenitor models as a function of the final mass. The filled triangles and circles denote the results of our binary star models at $Z=Z_{\odot}$ with $f_{\mathrm{WR}}=5$ and 10 , respectively. The filled squares give the results with the SMC metallicity models.

(A color version of this figure is available in the online journal.)

\subsection{Hydrogen}

In our binary star models, Case $\mathrm{AB}$ or Case $\mathrm{B}$ mass transfer does not completely remove hydrogen from the primary stars, as shown in Figure 4. The resulting WR mass loss is therefore weaker than that from the corresponding pure helium star. This explains the fact that for a given WR mass-loss rate, the binary star models with $M_{\mathrm{f}} \gtrsim 3.0 M_{\odot}$ predict somewhat larger $M_{\mathrm{He}}$ than the single helium star models do as shown in Figure 10.

More importantly, the binary star models show that, even at $Z \approx Z_{\odot}$, small amounts of hydrogen can be retained up to the pre-supernova stage for a certain range of the final mass. Figure 11 (see also Table 1 and Figure 4) shows the total mass of hydrogen in the primary stars at neon/oxygen burning phase. The time span from this stage to core collapse is supposed to be less than about $10 \mathrm{yr}$. The WR winds mass-loss rate from the primary star models shown in the figure is about $10^{-6} M_{\odot} \mathrm{yr}^{-1}$. Some of the primary stars in this sample are still undergoing Case ABB or BB mass transfer (Seqs. 5, 8, 9, 13, and 21 and many of the SMC models; see Table 1), but the mass transfer rate is only about $10^{-5} M_{\odot} \mathrm{yr}^{-1}$. Therefore, the amount of hydrogen at core collapse should remain close to the values given in the figure.

Figure 11 indicates that the presence of hydrogen is only expected for $3.0 \lesssim M_{\mathrm{f}}\left[M_{\odot}\right] \lesssim 3.7$, at solar metallicity with $f_{\mathrm{WR}}=5$. This results from two different reasons for different mass ranges. For $M_{\mathrm{f}}<3.0 M_{\odot}$, primary stars expand during the carbon burning phase to much larger radii than more massive ones do. The resulting mass-loss rates via Case ABB or BB transfer thus become large enough to completely remove hydrogen from the primary stars by the time of core collapse. For $M_{\mathrm{f}} \gtrsim 3.7 M_{\odot}$, on the other hand, WR winds are rather strong and can remove hydrogen from the primary stars during the core helium burning phase.

The upper limit of $M_{\mathrm{f}}$ for the presence of hydrogen thus

Figure 10. Amount of helium in SN Ibc progenitor models at $Z=Z_{\odot}$ with $f_{\mathrm{WR}}=5$, as function of the final mass. The filled circles denote the prediction from our binary star models. The results from mass-losing pure helium star models are marked by the dashed line.

(A color version of this figure is available in the online journal.) depends on the adopted mass-loss rate. As shown in the figure, with $f_{\mathrm{WR}}=10$, it increases to about $4.5 M_{\odot}$ at solar metallicity. At SMC metallicity, the stellar wind effect is less significant and 


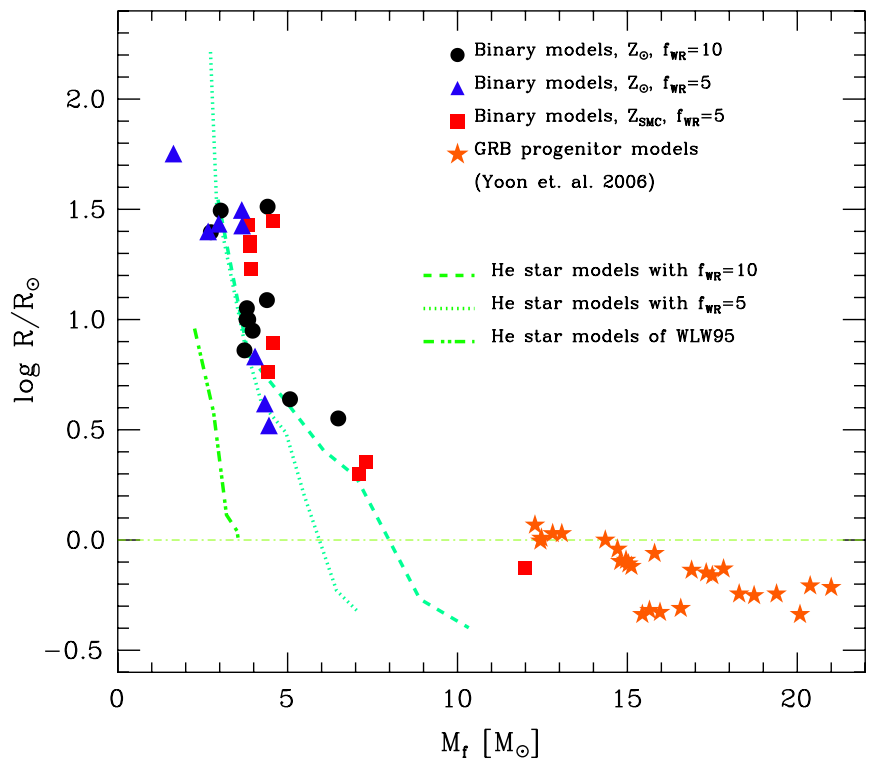

Figure 12. Predicted radii of SN Ibc progenitors as a function of the final mass. The filled circles and triangles denote the results of our binary star models at $Z=Z_{\odot}$ for $f_{\mathrm{WR}}=10$ and 5 , respectively, while the filled squares are for $Z=Z_{\text {SMC }}$. The results from mass-losing single helium star models at $Z=Z_{\odot}$ are marked by the dashed $\left(f_{\mathrm{WR}}=10\right)$, dotted $\left(f_{\mathrm{WR}}=5\right)$, and dashed-threedotted (WLW95) lines. The thin dash-dotted line gives the solar radius.

(A color version of this figure is available in the online journal.)

even rather massive supernova progenitors of about $7 M_{\odot}$ can retain fairly large amounts of hydrogen $\left(\sim 10^{-2} M_{\odot}\right)$. We discuss implications of this result for supernova types in Section 6.4.

\subsection{Radius}

Relatively low-mass helium stars usually experience a rapid expansion of the envelope during the core carbon burning phase. Interestingly, our new models predict much larger radii at the pre-supernova stages than what the WLW95 models do, as shown in Figure 12. The first reason for this difference is the updated OPAL opacity table by Iglesias \& Rogers (1996), which causes a strong iron bump at around $\log T=5.3$. Another reason is the smaller WR mass-loss rate adopted in the present study, and the resultant thicker helium envelope in our models. The radius is further affected by the complication of binary interaction: the presence of a thin hydrogen layer in some of the binary models results in a more extended envelope than in the corresponding single helium star models. It is also worth noting that the radii of the binary star models at $M_{\mathrm{f}}<3 M_{\odot}$ are smaller than those of the single helium star models. This is because the primary stars in these sequences are filling the Roche lobe, and the envelope cannot expand beyond it.

The metallicity effect is somewhat subtle. The hydrogen and helium layers in SN Ibc progenitors become thicker due to the reduced mass-loss rate for lower metallicity, while the opacity due to metals becomes smaller. As these two effects compensate each other, the radii of our SN Ibc progenitor models at both SMC and solar metallicities are found to be similar. Finally, it should be noted that the radius depends on the final mass. In general, a less massive progenitor tends to have a larger radius at the presupernova stage. This might make mixing of nickel into the helium rich layer induced by the Rayleigh-Taylor instability during the supernova explosion more efficient for a less massive SN Ibc progenitor, as implied by the simulations of Hachisu et al.



Figure 13. Predicted winds mass-loss rates of SN Ibc progenitors as a function of the final mass. The filled circles and triangles denote the results of our binary star models at $Z=Z_{\odot}$ for $f_{\mathrm{WR}}=10$ and 5 , respectively, while the filled squares are for $Z=Z_{\mathrm{SMC}}$. The results from mass-losing single helium star models at $Z=Z_{\odot}$ are marked by dashed $\left(f_{\mathrm{WR}}=10\right)$ and dotted $\left(f_{\mathrm{WR}}=5\right)$ lines.

(A color version of this figure is available in the online journal.)

(1991) and Joggerst et al. (2009), ${ }^{7}$ resulting in more prominent helium lines in the spectra (see Section 6).

\subsection{Winds}

Figure 13 shows the mass-loss rate due to WR winds at the central neon/oxygen burning phase from different SN Ibc progenitor models. The mass-loss rate ranges from $10^{-6} M_{\odot} \mathrm{yr}^{-1}$ to $\sim 10^{-5} M_{\odot} \mathrm{yr}^{-1}$, depending on the final mass, at solar metallicity. The corresponding wind velocity (i.e., the escape velocity) changes from $\sim 200 \mathrm{~km} \mathrm{~s}^{-1}$ to $\sim 2400 \mathrm{~km} \mathrm{~s}^{-1}$.

The circumstellar interaction of such winds from primary stars in binary systems is supposed to be very complex because of the orbital motion and the wind-wind interaction with the secondary star. Some of the primary star models shown in the figure are still undergoing Case $\mathrm{ABB}$ or $\mathrm{BB}$ mass transfer, which may add to the complexity. Therefore, the nature of the circumstellar materials around SN Ibc progenitors in a binary system may not follow the simple relation of $\rho \propto r^{-2}$ that is expected for single WR star progenitors.

\section{DISCUSSION}

We have presented new evolutionary models of massive close binary stars, considering tidal interaction, and transport of angular momentum and chemical species due to rotationally induced hydrodynamic instabilities and the Spruit-Tayler dynamo. We have investigated the redistribution of angular momentum in the primary star. Although mass transfer and tidal interaction can significantly affect the evolution of the rotation velocity of the primary star on the main sequence, the amount of angular momentum retained in the core in the late evolutionary stages is rather insensitive to the previous history of such binary interactions because of the self-regulating nature of the Spruit-Tayler dynamo.

We have also calculated non-rotating, mass-losing single helium star models and compared them with our primary star

\footnotetext{
7 However, it should be kept in mind that the degree of mixing due to the Rayleigh-Taylor instability may depend not only on the stellar structure but also on explosion energy and directional asymmetry (Hammer et al. 2010).
} 
models in binary systems. Our models adopt a much lower WR mass-loss rate than in the previous studies by WLW95 and WL99 and predict some new important properties of SN Ibc progenitors accordingly. The following discussions are based on the models with $f_{\mathrm{WR}}=5$, unless otherwise specified.

1. The final masses of SN Ibc progenitors in binary systems at $Z \simeq Z_{\odot}$ are not limited to $1.5 M_{\odot} \lesssim M_{\mathrm{f}} \lesssim 4 M_{\odot}$ as predicted by WLW95 and WL99, but a more wide range $M_{\mathrm{f}}$ is expected (i.e., $1.5 M_{\odot} \lesssim M_{\mathrm{f}} \lesssim 7.1 M_{\odot}$ from $M_{\text {init }} \simeq 12 \ldots 60 M_{\odot}$; see Figure 14$)$.

2. At $Z \simeq Z_{\odot}$, significant deficiency of helium $\left(M_{\mathrm{He}}<\right.$ $\left.0.5 M_{\odot}\right)$ is expected for $M_{\mathrm{f}} / M_{\odot} \gtrsim 5.5$ and for $1.5 \lesssim$ $M_{\mathrm{f}} / M_{\odot} \lesssim 2.0$ (Figure 10). Rather large amounts of helium up to $1.5 \widetilde{M}_{\odot}$ are expected for the other final mass range (i.e., $\left.2.0 \lesssim M_{\mathrm{f}} / M_{\odot} \lesssim 5.5 M_{\odot}\right)$. At $Z \simeq Z_{\mathrm{SMC}}$, no such helium deficient SN progenitors are expected for the considered initial masses $\left(16-40 M_{\odot}\right)$.

3. A thin layer of hydrogen with $M_{\mathrm{H}}=10^{-4}-10^{-2} M_{\odot}$ is predicted for $\mathrm{SN}$ Ibc progenitors with $3.0 M_{\odot} \lesssim M_{\mathrm{f}} \lesssim$ $3.7 M_{\odot}$ at $Z \simeq Z_{\odot}$ and $3.0 M_{\odot} \lesssim M_{\mathrm{f}} \lesssim 8 M_{\odot}$ at $Z \simeq$ $Z_{\mathrm{SMC}}$, respectively (Figure 11 , Table 1 ).

4. Most SN Ibc progenitors with $M_{\mathrm{f}} \lesssim 5 M_{\odot}$ rapidly expand during core carbon burning, resulting in $R=\sim 4.0-30 R_{\odot}$ at the presupernova stage. This is much larger than found in WLW95 (Figure 12). Compact progenitors of $R \lesssim R_{\odot}$ are only expected for a relatively high mass $\left(M_{\mathrm{f}} \gtrsim 5.5 M_{\odot}\right.$ at $Z \simeq Z_{\odot}$ and $M_{\mathrm{f}} \gtrsim 10 M_{\odot}$ at $Z \simeq Z_{\mathrm{SMC}}$; Figure 12 ).

The above results raise several important issues regarding observational consequences, as discussed below.

\subsection{Implications for Energetic Explosions Powered by Rapid Rotation}

Our binary star models show that the mass transfer during helium core contraction (Case AB or Case B) in a close massive binary system cannot remove the hydrogen envelope promptly enough to avoid the core braking due to the Spruit-Tayler dynamo during the helium core contraction phase. Comparison of our binary star models with the single star models by Heger et al. (2005) and Yoon et al. (2006) indicates that the amount of angular momentum retained in the core of the primary star at the presupernova stage should not be much different from those found in single star models if the Spruit-Tayler dynamo is adopted. That is, a specific angular momentum of a few $10^{14} \mathrm{~cm}^{2} \mathrm{~s}^{-1}$ in the innermost $\sim 1.4 M_{\odot}$ at the presupernova stage is expected in both single and binary stars. This value is smaller by 1 or 2 orders of magnitude than what is necessary to make long GRBs by magnetar or collapsar formation, or very energetic supernovae (hypernovae) powered by rapid rotation and strong magnetic fields (e.g., Burrows et al. 2007), although it may suffice to produce millisecond pulsars (Heger et al. 2005). Together with the work by Petrovic et al. (2005b), our results thus indicate that binary interactions with Case $A B / B$ mass transfers at $Z \approx Z_{\odot}$ may not particularly enhance the production of strongly rotation-powered events like long GRBs or hypernovae. This is consistent with the observational evidence that such events are rare compared to normal corecollapse events (e.g., Podsiadlowski et al. 2004; Guetta \& Della Valle 2007). This also confirms the theoretical consensus that other evolutionary paths are needed to produce long GRBs associated with SN Ibc, such as the quasi-chemically homogeneous evolution of a metal poor star (Yoon \& Langer 2005; Yoon et al. 2006; Woosley \& Heger 2006; Cantiello et al.
2007), tidal spin-up of a WR star in a very close binary system with a neutron star or BH companion (e.g., Izzard et al. 2004; van den Heuvel \& Yoon 2007), ${ }^{8}$ or binary evolution with Case C mass transfer with some specific conditions (Brown et al. 2000; Podsiadlowski et al. 2010).

\subsection{Progenitor Size}

Larger radii of our SN Ibc progenitor models than those previously found should have consequences in shock breakouts and bolometric light curves. For instance, a shock break-out from a larger envelope would be marked by a lower photospheric temperature. Detailed comparison of numerical calculations with observational data may thus give strong constraints on SNe Ibc progenitor properties (e.g., Calzavara \& Matzner 2004). Recent discovery of the X-ray outburst with SN 2008D by Soderberg et al. (2008) indeed suggests the usefulness of such a study for the probe of supernova progenitors (e.g., Soderberg et al. 2008; Chevalier \& Fransson 2008; Xu et al. 2008; Modjaz et al. 2009), for which our new models would provide ideal input. We will address this issue in a forthcoming paper.

\subsection{Presence of Helium and Implications for SNe Ic}

Although the weak signature or no evidence of helium in SNe Ic spectra may indicate the deficiency of helium in their progenitors, it is not well known how much helium can be hidden in the supernova spectra. It may also depend on the degree of mixing of nickel into helium rich layers (Woosley $\&$ Eastman 1995). If we assume $0.5 M_{\odot}$, for instance, as the maximum amount of helium allowed for hiding helium lines in SN spectra, our models indicate that most SNe Ic progenitors at solar metallicity should belong to two distinct classes in terms of both ZAMS and final masses, as summarized in Figure 14 for the binary systems that undergo Case B mass transfer. ${ }^{9}$ The same conclusion was also drawn by WL99 and Pols \& Dewi (2002). But Pols \& Dewi considered different types of binary systems (see below), and the finding of "two mass classes" for the final masses was not obvious in WL99 due to the very high WR rate adopted in their study, although it was clearly seen for the ZAMS masses.

If we assume that primary stars of $12.5 \lesssim M_{\text {ZAMS }} / M_{\odot} \lesssim$ 13.5 produce low-mass-class $\mathrm{SNe}$ Ic via Case $\mathrm{BB}$ mass transfer, about $62 \%$ of the $\mathrm{SNe}$ Ic from Case B systems should belong to the high mass class and the rest $(\sim 38 \%)$ to the low mass class, at $Z \approx Z_{\odot}$ (see Figure 14). It is important to note that the two classes are produced by different mechanisms. The high mass class of SNe Ic progenitors (i.e., $M_{\text {ZAMS }} \gtrsim 33 M_{\odot}$ in Figure 14) is a consequence of WR winds mass loss, while the low-massclass results from Case $\mathrm{ABB} / \mathrm{BB}$ mass transfer as discussed in Section 5.1. The ZAMS mass range for the low mass class may not be much affected by metallicity, while it should be widened with increasing metallicity for the high mass class. This leads to the conclusion that the low and high mass classes would dominate at low and high metallicities, respectively. It should also be noted that the final mass range for the low mass class may not change much for different metallicities, while it may decrease with increasing metallicity for the high mass class, due to the increasing WR winds mass-loss rates, as implied by the result of WL99.

\footnotetext{
8 A recent study using detailed stellar evolution models by Detmers et al. (2008), however, seriously questions this possibility.

9 For the systems with Case A mass transfer, each boundary should move to the right in the figure, but the parameter space explored with our model grid is not large enough to determine it quantitatively.
} 


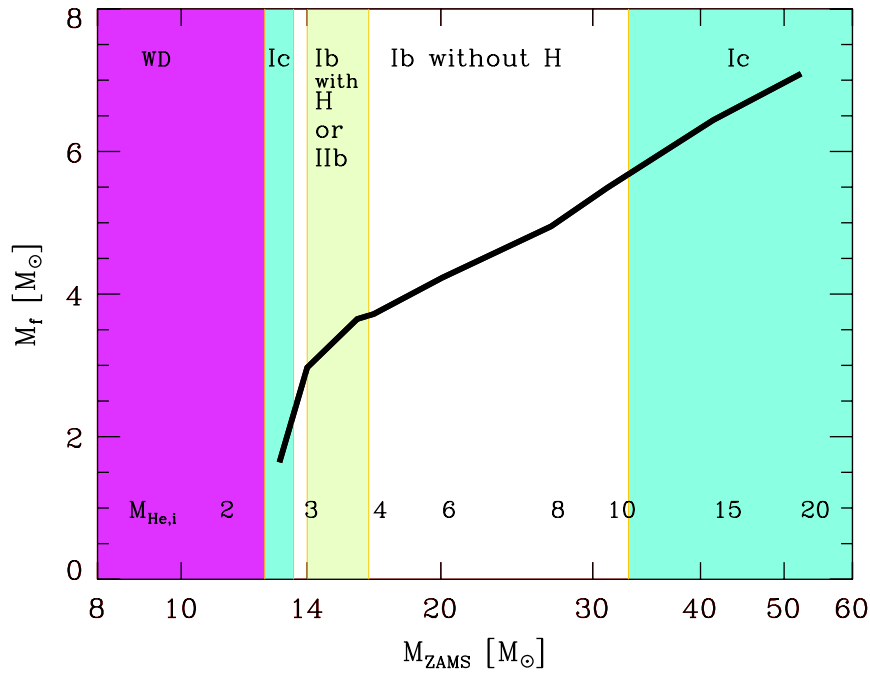

Figure 14. Predicted final masses of the primary stars in massive close binaries that undergo Case B mass transfer, as a function of the zero-age main sequence (ZAMS) mass, based on our binary and helium star models with $f_{\mathrm{WR}}=5$. The abscissa is given in log scale. The numbers right above the abscissa denote the initial masses of the helium stars that the primary stars of the corresponding ZAMS masses would produce. The expected final outcomes of the primary stars according to different ZAMS masses are given by the labels right below the top: white dwarf (WD), type Ic supernova (Ic), type Ib supernova with a thin hydrogen layer (Ib with $\mathrm{H}$ ) or type IIb supernova (IIb), and type Ib supernova without hydrogen (Ib without $\mathrm{H}$ ). Here we assumed $M_{\mathrm{He}}<0.5 M_{\odot}$ for SNe Ic progenitors. Note that the each boundary would shift to a higher value of $M_{\text {ZAMS }}$ for close binary systems with Case A mass transfer.

(A color version of this figure is available in the online journal.)

In the massive close binary systems considered in this paper, the primary star masses become much smaller than those of the secondary stars when Case $\mathrm{ABB}$ or Case $\mathrm{BB}$ mass transfer begins. If the companion star mass were lower than the helium star in a close binary system, the mass transfer rate should become higher than in the systems of the present study. For example, if a helium star is located in a very short period binary system $(P \lesssim 1$ day) with a less massive companion (e.g., a neutron star), mass transfer from the helium star may occur rapidly enough to make a helium-deficient carbon star, even for $M_{\mathrm{He}, \mathrm{i}} \approx 6.0 M_{\odot}$ as shown by Pols \& Dewi (2002), Dewi et al. (2002), and Ivanova et al. (2003). The final masses of such SN Ic progenitors may range from $1.5 M_{\odot}$ to $3.0 M_{\odot}$. This scenario was also suggested by Nomoto et al. (1994) to explain the fast light curve of Type Ic SN 1994I. The ZAMS mass of such a SN Ic should be in the range of 12-20 $M_{\odot}$ (Pols \& Dewi 2002). However, such close helium star plus neutron star systems are supposed to rarely form, and might not contribute much to the population of SNe Ic, compared to the systems considered in this study.

Our results should have several observational consequences. As mentioned above, the population of $\mathrm{SNe}$ Ic should be dominated by the high mass class for $Z \gtrsim Z_{\odot}$. They would have higher ZAMS and final masses than those of typical SNe Ib progenitors (cf. Kelly et al. 2008; Anderson \& James 2008). Given that the parameter space for the high-mass-class $\mathrm{SNe}$ Ic may become larger with a higher WR mass-loss rate, the number ratio of SNe Ic to $\mathrm{SNe} \mathrm{Ib}$, and that of high-massclass $\mathrm{SNe}$ Ic to low-mass-class SNe Ic should increase with increasing metallicity (cf. Prieto et al. 2008; Anderson \& James 2009; Boissier \& Prantzos 2009). The existence of the two mass classes of $\mathrm{SNe}$ Ic progenitors may be related to some aspects of the observational diversity of SNe Ic. For example, SNe Ic

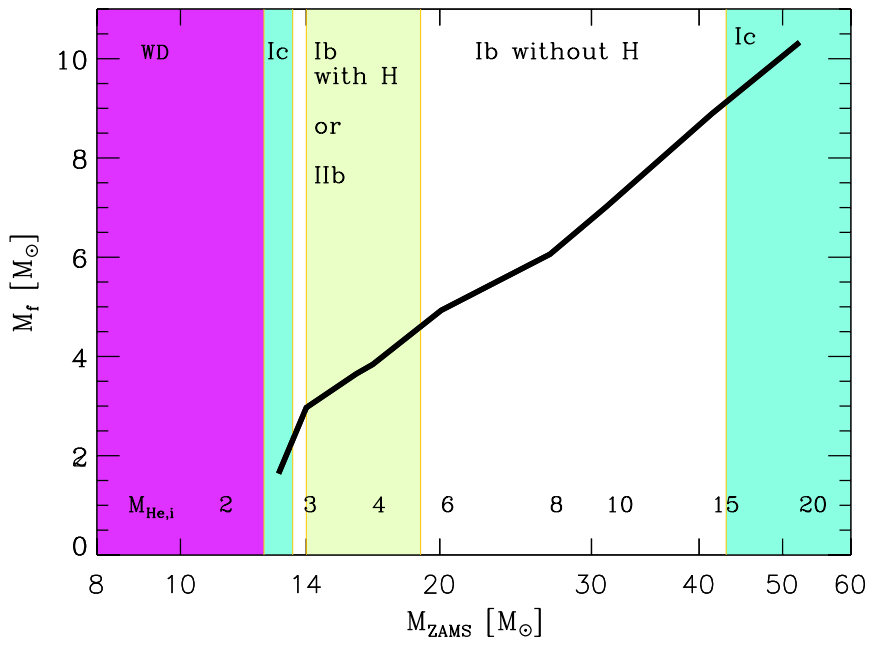

Figure 15. Same as in Figure 14, but with $f_{\mathrm{WR}}=10$.

(A color version of this figure is available in the online journal.)

of the low mass class is likely to be characterized by rather fast declining light curves and low luminosities (cf. Iwamoto et al. 1994; Richardson et al. 2006; Young et al. 2009), implying that the observed population of SNe Ic is likely to be biased to high-mass-class SNe Ic.

We should also note the huge difference of the binding energy between the two classes. The binding energy of the envelope above $1.4 M_{\odot}$ in the SN Ic progenitor star model of Seq. 3 $\left(M_{\mathrm{f}}=1.64 M_{\odot}\right)$ is only about $10^{49} \mathrm{erg}$, while it should be 1 or 2 orders of magnitude higher for a SN Ic progenitor with $M_{\mathrm{f}} \gtrsim 5.5 M_{\odot}$ (see Table 2). As a consequence, the energetics of SNe Ic might be systematically different for the two different classes.

On the other hand, the assumption of $M_{\mathrm{He}}<0.5 M_{\odot}$ for SN Ic progenitors leads to a ratio of type Ic to Ib supernova rate (Ic/Ib ratio) of about 0.4 from binary systems at solar metallicity. ${ }^{10}$ This appears in contradiction with recent observations that indicate rather a high Ic/Ib ratio of about 2.0 (e.g., Smartt 2009). This discrepancy would become even larger with $f_{\mathrm{WR}}=10$, as implied by Figure 15. This raises a question on the nature of SN Ic progenitors, and it should be kept in mind that we still do not fully understand what distinguishes SN Ic progenitors from those of SN Ib.

A recent work by Dessart et al. (2010) indicates that the mass fraction of helium in the outermost layers $\left(Y_{\mathrm{s}}\right)$, rather than the total mass of helium, may be more relevant for the presence of helium lines in supernova spectra. Specifically, it is shown that if helium is well mixed with $\mathrm{CO}$ material such that $Y_{\mathrm{s}}$ becomes less than about 0.5 , helium lines are not seen in early time spectra, despite a rather large total amount of helium $\left(M_{\mathrm{He}} \simeq 1.0 M_{\odot}\right)$, if non-thermal excitation is absent. In our progenitor models, such a small $Y_{\mathrm{S}}$ is realized only for $M_{\mathrm{f}} \gtrsim 5.5 M_{\odot}$ at solar metallicity (with $f_{\mathrm{WR}}=5$ ). This is not different from the above-discussed mass limit for having $M_{\mathrm{He}} \lesssim 0.5 M_{\odot}$, implying that the initial mass range for SN Ic

\footnotetext{
10 At SMC metallicity, the SN Ibc progenitor models of initial masses of 16-40 $M_{\odot}$ have $3.9 \lesssim M_{\mathrm{f}} \lesssim 12 M_{\odot}$ with $M_{\mathrm{He}} \gtrsim 1.2 M_{\odot}$ (see Table 1$)$. This implies that the low-mass-class SNe Ic would predominantly occur in binary systems at this metallicity. If we assume stars with $12.5 \lesssim M_{\text {init }} \lesssim 13.5$ would produce low-mass-class SNe Ic as in the case of solar metallicity, the $\mathrm{SN}$ Ic/SN Ib ratio would be about 0.1 at SMC metallicity. The exploration of the exact mass range for the low-mass-class SNe Ic is a time-consuming task, and we plan to investigate this in the near future.
} 
progenitors would not change much even if we adopted $Y_{\mathrm{s}}$ as a criterion, at least for the high mass class. On the other hand, we have $Y_{\mathrm{s}}=0.98$ in the primary star of Seq. 3 at carbon exhaustion while the total mass of helium is less than $0.2 M_{\odot}$. This implies that the initial mass range for the low-mass-class SN Ic progenitors might be affected if the condition of $Y_{\mathrm{S}}<0.5$ for SN Ic progenitors were applied. But Dessart et al. (2010) did not yet calculate such a low-mass SN progenitor model $\left(M_{\mathrm{f}} \lesssim 2.0 M_{\odot}\right)$, and their analyses were limited to early times of supernovae. It remains to be an important subject of future work to systematically investigate which types of supernova progenitors would lead to the presence or absence of helium lines in the supernova spectra at different epochs, including the effect of non-thermal excitation. Therefore, the above discussion on Type Ic progenitors based on the total amount of helium should only be considered indicative at this stage.

\subsection{Presence of Hydrogen}

It is interesting that, at $Z \approx Z_{\odot}$, the presence of a thin hydrogen layer is only expected for a limited range of the initial/ final mass of SN Ib progenitors, as shown in Figures 11 and 14. The detection of hydrogen absorption lines at high velocity has been indeed reported in many SNe Ib (e.g., Deng et al. 2000; Branch et al. 2002; Elmhamdi et al. 2006), in favor of our model prediction for the presence of a thin hydrogen layer in $\mathrm{SNe}$ Ib progenitors. This might provide a strong constraint for the progenitor masses of observed $\mathrm{SNe} \mathrm{Ib}$, in principle.

Note also that explosions of such helium stars with thin hydrogen layers could be recognized as SN IIb rather than Ib, if hydrogen lines were detected shortly after supernova explosion, e.g., as in the case of SN 2008ax (Chornock et al. 2010) and as recently discussed by Spencer \& Baron (2010) and Dessart et al. (2010). The radii of these progenitor models range from $\sim 10^{11} \mathrm{~cm}$ to $\sim 10^{12} \mathrm{~cm}$. They may correspond to the "compact" category of SN IIb progenitors, which is discussed in Chevalier \& Soderberg (2010). The relatively low ejecta masses of such $\mathrm{SNe}$ IIb are consistent with our model predictions.

On the other hand, Case C mass transfer can also leave helium cores covered with small amounts of hydrogen envelope. As the lifetime of such stars made via Case $\mathrm{C}$ mass transfer should be rather short, they can retain much more hydrogen $\left(M_{\mathrm{H}}>0.1 M_{\odot}\right)$, than what is predicted from our binary models with Case $\mathrm{AB} / \mathrm{B}$ mass transfer. Such a star may eventually explode as a SN IIb like SN 1993J (e.g., Podsiadlowski et al. 1993; Maund et al. 2004), with a much extended envelope $\left(\sim 10^{13}-10^{14} \mathrm{~cm}\right)$.

Therefore, the two categories of SN IIb progenitors according to their sizes, which has been recently suggested by Chevalier \& Soderberg (2010), may be understood within the framework of binary evolution; $\mathrm{SNe}$ IIb of the compact type may be produced via Case $\mathrm{AB} / \mathrm{B}$ mass transfer (especially at $Z \lesssim Z_{\odot}$ ), and $\mathrm{SNe}$ IIb of the extended type via Case $\mathrm{C}$ mass transfer.

This work is supported by the DOE SciDAC Program (DOE DE-FC02-06ER41438), the NSF grant (NSF-ARRA AST0909129), and the NASA Theory Program (NNX09AK36G). We are grateful to Luc Dessart for useful discussions.

\section{REFERENCES}

Anderson, J. P., \& James, P. A. 2008, MNRAS, 390, 1527

Anderson, J. P., \& James, P. A. 2009, MNRAS, 399, 559

Boissier, S., \& Prantzos, N. 2009, A\&A, 503, 137
Branch, D., et al. 2002, ApJ, 566, 1005

Braun, H., \& Langer, N. 1995, A\&A, 297, 483

Brookshaw, L., \& Tavani, M. 1993, ApJ, 410, 719

Brown, G. E., Lee, C.-H., Wijers, R. A. M. J., Lee, H. K., Istraelian, G., \& Bethe, H. A. 2000, New Astron., 5, 191

Burrows, A., Dessart, L., Livne, E., Ott, C. D., \& Murphy, J. 2007, ApJ, 664, 416

Calzavara, A. J., \& Matzner, C. D. 2004, MNRAS, 351, 694

Cantiello, M., Yoon, S.-C., Langer, N., \& Livio, M. 2007, A\&A, 465, 29

Chevalier, R. A., \& Fransson, C. 2008, ApJ, 683, 135

Chevelier, R. A., \& Soderberg, 2010, ApJ, 711, L40

Chornock, R., et al. 2010, arXiv:1001.2775

de Donder, E., \& Vanbeveren, D. 1998, A\&A, 333, 557

Deng, J. S., Qiu, Y. L., Hu, J. Y., Hatano, K., \& Branch, D. 2000, ApJ, 540, 452

Denissenkov, P. A., \& Pinsonneault, M. 2007, ApJ, 655, 1157

Dessart, L., Hillier, D. J., Livne, E., Yoon, S.-C., Woosley, S. E., Walman, R., \& Langer, N. 2010, MNRAS, submitted

Detmers, R. G., Langer, N., Podsiadlowski, Ph., \& Izzard, R. G. 2008, A\&A, 484,831

Dewi, J. D. M., Pols, O. R., Savonije, G. J., \& van den Heuvel, E. P. J. 2002, MNRAS, 331, 1027

Eggleton, P. 1983, ApJ, 263, 368

Eldridge, J. J., Izzard, R. G., \& Tout, C. A. 2008, MNRAS, 384, 1109

Eldridge, J. J., \& Vink, J. S. 2006, A\&A, 452, 295

Elmhamdi, A., Danziger, I. J., Branch, D., Leibundgut, B., Baron, E., \& Kirshner, R. P. 2006, A\&A, 450, 305

Endal, A., \& Sofia, S. 1976, ApJ, 210, 184

Fryer, C. L. 1999, ApJ, 522, 413

Georgy, C., Meynet, G., Walder, R., Folini, D., \& Maeder, A. 2009, A\&A, 502, 611

Gräfener, G., \& Hamann, W.-R. 2008, A\&A, 482, 945

Guetta, D., \& Della Valle, M. 2007, ApJ, 657, 73

Hachisu, I., Matsuda, T., Nomoto, K., \& Shigeyama, T. 1991, ApJ, 368, 27

Hamann, W.-R., \& Koesterke, L. 1998, A\&A, 335, 1003

Hamann, W.-R., Koesterke, L., \& Wessolowski, U. 1995, A\&A, 299, 151

Hamann, W.-R., Schönberner, D., \& Heger, U. 1982, A\&A, 116, 273

Hammer, N. J., Janka, H.-Th., \& Mueller, E. 2010, ApJ, 714, 1371

Heger, A., \& Langer, N. 2000, ApJ, 544, 1016

Heger, A., Langer, N., \& Woosley, S. E. 2000, ApJ, 528, 368

Heger, A., Woosley, S. E., \& Spruit, H. C. 2005, ApJ, 626, 350

Hirschi, R., Meynet, G., \& Maeder, A. 2004, A\&A, 425, 649

Iglesias, C. A., \& Rogers, F. J. 1996, ApJ, 464, 943

Ivanova, N., Belczynski, K., Kalogera, V., Rasio, F. A., \& Taam, R. E. 2003, ApJ, 592,475

Iwamoto, K., Nomoto, K., Höflich, P., Yamaoka, H., Kumagai, S., \& Shigeyama, T. 1994, ApJ, 437, L115

Izzard, R. G., Ramirez-Ruiz, E., \& Tout, C. A. 2004, MNRAS, 348 , 1215

Joggerst, C. C., Woosley, S. E., \& Heger, A. 2009, ApJ, 693, 1780

Kelly, P. L., Kirshner, R. P., \& Pahre, M. 2008, ApJ, 687, 1201

Kudritzki, R. P., Pauldrach, A., Puls, J., \& Abbott, D. C. 1989, A\&A, 219, 205

Langer, N. 1989, A\&A, 220, 135

Langer, N. 1998, A\&A, 329, 551

Langer, N. 2009, A\&A, 500, 133

Langer, N., Fricke, K. J., \& Sugimoto, 1983, A\&A, 126, 207

Langer, N., Wellstein, S., \& Petrovic, J. 2003, in IAU Symp. 212, A Massive Star Odyssey: From Main Sequence to Supernova, ed. K. van der Hucht, A. Herrero, \& E. César (Cambridge: Cambridge Univ. Press), 275

Limongi, M., \& Chieffi, A. 2006, ApJ, 647, 483

Maund, J. R., Smartt, S. J., Kudritzki, R. P., Podsiadlowski, Ph., \& Gilmore, G. F. 2004, Nature, 427, 129

Maeder, A., \& Meynet, G. 2000, ARA\&A, 38, 143

Meynet, G., \& Maeder, A. 1997, A\&A, 321, 465

Meynet, G., \& Maeder, A. 2003, A\&A, 404, 975

Meynet, G., \& Maeder, A. 2005, A\&A, 429, 581

Modjaz, M., et al. 2009, ApJ, 702, 226

Nomoto, K., Yamaoka, H., Pols, O. R., van den Heuvel, E. P. J., Iwamoto, K., Kumagai, S., \& Shigeyama, T. 1994, Nature, 371, 227

Nugis, T., \& Lamers, H. J. G. L. M. 2000, A\&A, 360, 227

Petrovic, J., Langer, N., \& van der Hucht, K. A. 2005a, A\&A, 435, 1013

Petrovic, J., Langer, N., Yoon, S.-C., \& Heger, A. 2005b, A\&A, 435, 247

Podsiadlowski, Ph., Hsu, J. J. L., Joss, P. C., \& Ross, R. R. 1993, Nature, 364 509

Podsiadlowski, Ph., Ivanova, N., Justham, S., \& Rappaport, S. 2010, MNRAS, 406, 840 
Podsiadlowski, Ph., Joss, P. C., \& Hsu, J. J. L. 1992, ApJ, 391, 246

Podsiadlowski, Ph., Mazzali, P. A., Nomoto, K., Lazzati, D., \& Cappellaro, E. 2004, ApJ, 607, 17

Pols, O. R., \& Dewi, D. M. 2002, PASA, 19, 233

Prieto, J. L., Stanek, K. Z., \& Beacom, J. F. 2008, ApJ, 673, 999

Richardson, D., Branch, D., \& Baron, E. 2006, ApJ, 131, 2233

Ritter, H. 1988, A\&A, 202, 93

Smartt, S. J. 2008, ARA\&A, 47, 63

Soderberg, A. M., et al. 2008, Nature, 453, 469

Spencer, J., \& Baron, E. 2010, ApJ, 718, 957

Spruit, H. C. 1999, A\&A, 349, 189

Spruit, H. C. 2002, A\&A, 381, 923

Suijs, M. P. L., Langer, N., Poelarends, A.-J., Yoon, S.-C., Heger, A., \& Herwig, F. 2008, A\&A, 481, 87

Tassoul, J.-L. 1987, ApJ, 322, 856

Tassoul, J.-L. 2000, Stellar Rotation (Cambridge: Cambridge Univ. Press)

Toledano, O., Moreno, E., Koenigsberger, G., Detmers, R., \& Langer, N. 2007, A\&A, 461, 1057

van den Heuvel, E. P. J., \& Yoon, S.-C. 2007, Ap\&SS, 311, 177
Vink, J. S., \& de Koter, A. 2005, A\&A, 442, 587

Vink, J. S., de Koter, A., \& Lamers, H. J. G. L. M. 2001, A\&A, 369, 574

Wellstein, S. 2001, PhD thesis, Potsdam Univ.

Wellstein, S., \& Langer, N. 1999, A\&A, 350, 148 (WL99)

Wellstein, S., Langer, N., \& Braun, H. 2001, A\&A, 369, 939

Woosley, S. E. 1993, ApJ, 405, 273

Woosley, S. E., \& Bloom, J. S. 2006, ARA\&A, 44, 507

Woosley, S. E., \& Eastman, R. G. 1995, in Thermonuclear Supernovae, ed. P. Ruiz-LaPuente, R. Canal, \& J. Isern (NATO ASI Ser. C., Vol. 486; Dordrecht: Kluwer)

Woosley, S. E., \& Heger, 2006, ApJ, 637, 914

Woosley, S. E., Langer, N., \& Weaver, 1993, ApJ, 411, 823

Woosley, S. E., Langer, N., \& Weaver, 1995, ApJ, 448, 315 (WLW95)

Xu, D., Zou, Y.-C., \& Fan, Y.-Z. 2008, arXiv:0801.4325

Yoon, S.-C., \& Langer, N. 2005, A\&A, 443, 643

Yoon, S.-C., Langer, N., \& Norman, C. 2006, A\&A, 460, 199

Young, D. R., et al. 2009, A\&A, 512, 70

Zahn, J.-P. 1977, A\&A, 57, 383

Zahn, J.-P., Brun, A. S., \& Mathis, S. 2007, A\&A, 474, 145 